\title{
Groups, Jacobi functions and rigged Hilbert spaces
}

\author{
E. Celeghini \& * \\ Dipartimento di Fisica, Università di Firenze \\ and INFN-Sezione di Firenze \\ 150019 Sesto Fiorentino, Firenze, Italy. \\ M. Gadelld and M. A. del Olmof \\ \& Departamento de Física Teórica, Atómica y Óptica, \\ IMUVA - Mathematical Research Institute, \\ Universidad de Valladolid, E-47005 Valladolid, Spain.
}

(Dated: November 21, 2019)

\begin{abstract}
This paper is a contribution to the study of the relations between special functions, Lie algebras and rigged Hilbert spaces. The discrete indices and continuous variables of special functions are in correspondence with the representations of their algebra of symmetry, that induce discrete and continuous bases coexisting on a rigged Hilbert space supporting the representation. Meaningful operators are shown to be continuous on the spaces of test vectors and its dual. Here, the chosen special functions, called "Algebraic Jacobi Functions" are related to the Jacobi polynomials and the Lie algebra is $s u(2,2)$. These functions with $m$ and $q$ fixed, also exhibit a $s u(1,1)$-symmetry. Different discrete and continuous bases are introduced. An extension in the spirit of the associated Legendre polynomials and the spherical harmonics is presented introducing the "Jacobi Harmonics" that are a generalization of the spherical harmonics to the three-dimensional hypersphere $S^{3}$.
\end{abstract}

PACS numbers: 02.20.Sv, 02.30.Gp, 02.30.Px, 02.30.Tb, 03.65.-w, 03.65.Ca

Keywords: Special functions, Jacobi polynomials, Lie groups and algebras, rigged Hilbert spaces, harmonic analysis

\section{INTRODUCTION}

This article belongs to a series of papers intended to give a unified picture of the connections between Lie algebras, special functions and rigged Hilbert spaces. The latter share with the related Lie algebras a common property, which is the need to deal simultaneously with operators, with discrete and continuous spectrum and with their eigenvectors while special functions depend on discrete indices and on continuous variables. All of them have thus a common general frame.

One of the main interests of this line of research is to give a consistent description of Quantum Mechanics, that demand the coexistence of operators with discrete spectrum (like the Hamiltonian for the harmonic oscillator) and continuous spectrum (like the position and momentum operators, etc.), along to their eigenvalues.

In previous works, we have obtained the algebraic structure associated to some orthogonal polynomials such as Hermite, Legendre, Laguerre, associ-

\footnotetext{
* celeghini@fi.infn.it

† manuelgadella1@gmail.com

¥ marianoantonio.olmo@uva.es
}

ated Laguerre polynomials or spherical harmonics [1 4]. Each class of orthogonal polynomials is associated to a particular representation of a specific Lie group. This is the case of Hermite polynomials with Heisenberg-Weyl group or associated Legendre polynomials and spherical harmonics with $S O(3,2)$. Spaces supporting the representation of these associated groups include discrete as well as continuous basis as eigenvectors of the Lie algebra generators or some functions constructed with these generators. Hilbert spaces are not sufficient to bear a representation of these groups and their corresponding algebras, as they do not have continuous bases. These bases are quite often used by physicists, so that it would be convenient to have representation spaces including both discrete and continuous basis. For this reason, we move to rigged Hilbert spaces where different cardinalities find a formal arrangement.

A rigged Hilbert space (RHS) is a triplet of spaces

$$
\Psi \subset \mathcal{H} \subset \Psi^{\times},
$$

where, $\mathcal{H}$ is an infinite dimensional separable Hilbert space. The space $\Psi$ is a dense subspace endowed with a locally convex topology (in many cases produced by an infinite set of norms that include the Hilbert space norm) stronger than the 
topology induced by the Hilbert space topology (the canonical injection is continuous). It is sometimes called the space of test vectors or even the test space. The space $\Psi^{\times}$is the (anti)-dual space of $\Psi$, i.e., the space of all (anti)-linear continuous functionals on $\Psi$, endowed with a topology compatible with the dual pair structure (quite often the weak topology). The action of $F \in \Psi^{\times}$on each vector $\varphi \in \Psi$ gives a complex number often denoted as $\langle\varphi \mid F\rangle$, following the Dirac notation.

It is not our intention here to review properties and applications of RHS, the reader may visit for this purpose the extensive literature on the subject [5-13], but we would like to mention that recently they have received renewed attention, see 14 - 19$]$, including their relations with partial inner products 20] and frames 21]. We limit us to remember that our primary purpose is to provide of mathematical sense to the Dirac formulation of quantum mechanics and its continuous basis of eigenvectors of observables. Also, it is important to recall that if $\mathcal{O}$ is an operator on $\mathcal{H}$, such that $\Psi$ reduces its adjoint operator $\mathcal{O}^{\dagger}$ (i.e., $\mathcal{O}^{\dagger} \Psi \subset \Psi$ ) and $\mathcal{O}^{\dagger}$ is continuous with the topology on $\Psi$, then, it is possible to extend $\mathcal{O}$ into $\Psi^{\times}$by means of the duality formula

$$
\left\langle\mathcal{O}^{\dagger} \varphi \mid F\right\rangle=\langle\varphi \mid \hat{\mathcal{O}} F\rangle, \quad \forall \varphi \in \Psi, \quad \forall F \in \Psi^{\times} .
$$

This expression is also valid if $\mathcal{O}$ is self-adjoint. Hereafter for the sake of the simplicity we will also denote by the same symbol the operator $(\mathcal{O})$ and its extension $(\hat{\mathcal{O}})$.

Two RHS $\Psi \subset \mathcal{H} \subset \Psi^{\times}$and $\Phi \subset \mathcal{L} \subset \Phi^{\times}$are unitarily equivalent if there is a unitary mapping $U: \mathcal{H} \longmapsto \mathcal{L}$ such that $U \Psi=\Phi$ is a one-to-one onto mapping preserving topologies, i.e., $U$ and its inverse, $U^{-1}$, are continuous. As a consequence, the duality formula (10)

$$
\langle U \varphi \mid U F\rangle=\langle\varphi \mid F\rangle, \quad \forall \varphi \in \Psi, \quad \forall F \in \Psi^{\times},
$$

extends $U$ to a continuous one-to-one mapping from $\Psi^{\times}$onto $\Phi^{\times}$with continuous inverse, i.e. we have the diagram

$$
\begin{array}{rrrrr}
\Psi & \subset \mathcal{H} & \subset & \Psi^{\times} \\
U \downarrow & U \downarrow & U \downarrow \\
\Phi & \subset & \mathcal{L} & \subset \Phi^{\times}
\end{array} .
$$

A typical situation comes when the former is an abstract RHS and the other is a space in which $\Phi$ and $\mathcal{L}$ are spaces of functions. We say that the second is a representation of the former.
In previous articles, we have analysed the connections between Hermite and Laguerre functions with the fractional Fourier transformation and signal theory 22], discrete and continuous basis for the representations of $S O(3,2)$ and the role of spherical harmonics [23], and similar discussions concerning other groups and their algebras such as $S O(2)$ [24], some considerations on harmonic analysis on the circle 25], or $S U(2)$, associated Laguerre polynomials 26] and Zernike polynomials, used in optics, related to the group $S U(1,1) \otimes S U(1,1)$ [27]. In Ref. 28] the interested reader can find a review devoted to the relation between Lie groups, special functions and rigged Hilbert spaces.

From the formal point of view, operators with purely discrete spectrum are connected to discrete indices of special functions. They are continuous on certain test space of fuctions $\Phi$ of a given RHS and determine the unitary irreducible representations (UIR) of Lie algebras. Analogously, operators with continuous spectrum are related with the dependence of special functions with respect to continuous real variables. They are continuous on $\Phi$ and, therefore, may be extended by continuity to the anti-dual space $\Phi^{\times}$, where their eigenvectors are well defined. Recall that eigenvectors for self-adjoint operators corresponding to eigenvalues in the continuous spectrum are not normalizable. In this paper, we propose a detailed study of the "Algebraic Jacobi Functions" related to the Jacobi polynomials, that we introduced in [3, 29]. The formal structure underlying Jacobi polynomials is discussed. In particular, we analyse topological properties of the relevant ladder operators, including the generators of the Lie algebra. The motivation of our work is twofold. Firstly, as we mention before, we have studied other orthogonal polynomials, like Hermite, Laguerre, Legendre, Associate Legendre, Spherical Harmonics and all of them are related to the Jacobi polynomials [1, 2]. On the other hand, the Jacobi polynomials are involved in many applications in different science areas. For instance, in physics in the study of quantum superintegrable systems as solutions of such as systems 32, 33], in the study of the rotations on the the space through the $d_{j}$-Wigner rotation matrices [34, 35], in signal processing like in the study of solutions of transverse vibrations of nonuniform homogeneous beams and plates [36], or in the data compression of electrocardiograms [37] in the medicine field or properly in mathematics as approximated solutions of singular integral equations 38], in numerical solutions of integro-differential- 
difference equations [39], and in the obtention of Jacobi approximations in Hilbert spaces to solve singular differential equations [40], or in spectral methods for solving partial differential equations on unbounded domains [41].

The paper is organized as follows. Next Section presents a brief revision of known material: the Algebraic Jacobi Functions together with some of their relevant properties and their symmetries, the Lie algebra $s u(2,2)$. In Section [II] we introduce an infinity family of rigged Hilbert spaces $\left\{\Phi_{m, q} \subset \mathcal{L}_{m, q} \subset\left(\Phi_{m, q}\right)^{\times}\right\}$labelled by two (fixed) integer or half-integer indices $(m, q)$ where $\mathcal{L}_{m, q}=$ $L^{2}[-1,1]$ and we study the operators acting inside $\Phi_{m, q}$ and into different spaces $\Phi_{m, q}$ all of them belonging to $s u(2,2)$ or to its Universal Enveloping Algebra UEA $[s u(2,2)]$. It is proved that these operators are continuous and can be extended continuously to the dual spaces. The discrete and continuous bases are studied in Section IV For that purpose an abstract Hilbert space $\mathcal{H}_{m, q}$ is introduced via a unitary map, $U$, from $\mathcal{H}_{m, q}$ into $\mathcal{L}_{m, q}$. In this way we obtain a new family of abstract rigged Hilbert spaces $\left(\Psi_{m, q} \subset\right.$ $\left.\mathcal{H}_{m, q} \subset\left(\Psi_{m, q}\right)^{\times}\right)$unitarily equivalent to the former one, In this new family we introduce a discrete basis $\left(\{|j,(m, q)\rangle\}_{j \geq \sup (|m|,|q|)}^{\infty}\right)$, while a discrete- continuous basis $\left(\{|x,(m, q)\rangle\}_{x \in[-1,1]}\right)$ is associated with each element of the original family of RHS. Also the $s u(2,2)$ operators are translated to the new RHS as well as their properties by means of $U$. Section $\nabla$ is devoted to consider all together the AJF in a "total space" with a inner product, for that we extend the AJF following the procedure used with the Associated Legendre polynomials to obtain the Spherical Harmonics, but here we have to consider two angular variables, $\theta$ and $\xi$ associated the discrete labels $m$ and $q$, respectively, and not only one like in the Spherical Harmonics. We also prove that the $s u(2,2)$ operators are continuous and also continuously extended to the dual. Moreover we obtain two RHS one associated to the integer values of $(j, m, q)$ and the other one for the half-integer values. After a section of conclusions we have added three appendices.

\section{ALGEBRAIC JACOBI FUNCTIONS: AN OVERVIEW}

The Jacobi polynomials of order $n \in \mathbb{N}, J_{n}^{\alpha, \beta}(x)$, are usually defined as 42,43

$$
J_{n}^{(\alpha, \beta)}(x):=\sum_{s=0}^{n} \frac{\Gamma(n+\alpha+1)}{\Gamma(s+1) \Gamma(n+\alpha-s+1)} \frac{\Gamma(n+\beta+1)}{\Gamma(n-s+1) \Gamma(\beta+s+1)}\left(\frac{x+1}{2}\right)^{s}\left(\frac{x-1}{2}\right)^{n-s} .
$$

They verify the following second order differential equation

$$
\left[\left(1-x^{2}\right) \frac{d^{2}}{d x^{2}}-((\alpha+\beta+2) x+(\alpha-\beta)) \frac{d}{d x}+n(n+\alpha+\beta+1)\right] J_{n}^{(\alpha, \beta)}(x)=0 .
$$

\section{A. Algebraic Jacobi Functions}

We now consider the representation spaces of the Lie algebras $s u(2,2)$ and $s u(1,1)$ that have as bases the Algebraic Jacobi Functions (AJF) defined in terms of the Jacobi polynomials $J_{n}^{(\alpha, \beta)}(x)$ by [3]:

$$
\mathcal{J}_{j}^{m, q}(x):=\sqrt{\frac{\Gamma(j+m+1) \Gamma(j-m+1)}{\Gamma(j+q+1) \Gamma(j-q+1)}}\left(\frac{1-x}{2}\right)^{\frac{m+q}{2}}\left(\frac{1+x}{2}\right)^{\frac{m-q}{2}} J_{j-m}^{(m+q, m-q)}(x),
$$

where

$$
j:=n+\frac{\alpha+\beta}{2}, \quad m:=\frac{\alpha+\beta}{2}, \quad q:=\frac{\alpha-\beta}{2} .
$$

or

$$
n=j-m, \quad \alpha=m+q, \quad \beta=m-q,
$$


Considerations derived from the theory of group representations force the following restrictions in the above parameters:

$$
j \geq|m|, \quad j \geq|q|, \quad 2 j \in \mathbb{N}, \quad j-m \in \mathbb{N}, \quad j-q \in \mathbb{N},
$$

with the parameters $(j, m, q)$ all together integers or half-integers. Conditions (5) in terms of the original parameters $(n, \alpha, \beta)$ are

$$
n \in \mathbb{N}, \quad \alpha, \beta \in \mathbb{Z}, \quad \alpha, \beta \geq-n, \quad \alpha+\beta \geq-n
$$

The values of $\mathcal{J}_{j}^{m, q}(x)$ at the points $x= \pm 1$ are defined by means of the appropriate limits. In Appendix A it is shown that $\mathcal{J}_{j}^{m, q}(x)$ are real polynomials or "quasi-polynomials" for $-1 \leq x \leq 1$ and that they have quite more symmetries than the Jacobi polynomials, i.e.

$$
\mathcal{J}_{j}^{m, q}(x)=\mathcal{J}_{j}^{q, m}(x)=(-1)^{m+q} \mathcal{J}_{j}^{-m,-q}(x)=(-1)^{j-m} \mathcal{J}_{j}^{m,-q}(-x)
$$

The Algebraic Jacobi Functions $\mathcal{J}_{j}^{m, q}(x)$ verify the differential equation

$$
\left[-\left(1-x^{2}\right) \frac{d^{2}}{d x^{2}}+2 x \frac{d}{d x}+\frac{2 m q x+m^{2}+q^{2}}{1-x^{2}}-j(j+1)\right] \mathcal{J}_{j}^{m, q}(x)=0,
$$

In addition, for fixed $m$ and $q$ the AJF satisfy the following relations:

$$
\int_{-1}^{1} \mathcal{J}_{j}^{m, q}(x)(j+1 / 2) \mathcal{J}_{j^{\prime}}^{m, q}(x) d x=\delta_{j, j^{\prime}}
$$

and

$$
\sum_{j \geq \sup (|m|,|q|)}^{\infty} \mathcal{J}_{j}^{m, q}(x)(j+1 / 2) \mathcal{J}_{j}^{m, q}(y)=\delta(x-y) .
$$

In expressions (7) and (8) $j, m, q$ are all together integrer or half-integer and satisfy (5). This shows that for fixed $m$ and $q$ both in- teger or half-integer, the set of AJF given by $\left\{J_{j}^{m, q}(x)\right\}_{j \geq \sup (|m|,|q|)}^{\infty}$ forms an orthogonal basis of the Hilbert space $L^{2}[-1,1]$. However, the multiplication for $\sqrt{j+1 / 2}$ of the $\operatorname{AJF} \mathcal{J}_{j}^{m, q}(x)$ allows to obtain the related orthonormal basis of $L^{2}[-1,1]$ in the framework of RHS. In the following we shall use AJF to discuss the symmetry operators and the Normalized Algebraic Jacobi Functions (NAJF)

$$
\mathbb{J}_{j}^{m, q}(x):=\sqrt{j+1 / 2} \mathcal{J}_{j}^{m, q}(x)
$$

as orthonormal basis. The relations (77) and (8) become

$$
\begin{aligned}
\left\langle\mathbb{J}_{j}^{m, q}(x) \mid \mathbb{J}_{j^{\prime}}^{m, q}(x)\right\rangle & \left.\equiv \int_{-1}^{1} \mathbb{J}_{j}^{m, q}(x)\right) \mathbb{J}_{j^{\prime}}^{m, q}(x) d x=\delta_{j, j^{\prime}}, \\
\sum_{j \geq \sup (|m|,|q|)}^{\infty}\left|\mathbb{J}_{j}^{m, q}(x)\right\rangle\left\langle\mathbb{J}_{j}^{m, q}(y)\right| & \equiv \sum_{j \geq \sup (|m|,|q|)}^{\infty} \mathbb{J}_{j}^{m, q}(x) \mathbb{J}_{j}^{m, q}(y)=\delta(x-y) .
\end{aligned}
$$

Note that the AJF have a direct relation with the Legendre functions:

$$
P_{l}(x)=\mathcal{J}_{l}^{0,0}(x), \quad P_{l}^{m}(x)=(-1)^{m} \sqrt{\frac{(l+m) !}{(l-m) !}} \mathcal{J}_{l}^{m, 0}(x),
$$

as can be obtained from [30] and [31] (p. 213). 


\section{B. $s u(2,2)$ and $s u(1,1)$ symmetry algebras on AJF}

The explicit formulae for the ladder operators, $A_{ \pm}, B_{ \pm}, C_{ \pm}, D_{ \pm}, E_{ \pm}, F_{ \pm}$on the Algebraic Jacobi Functions are given in [3, 29]. They are generators of the $s u(2,2)$ Lie algebra and their action on the AJF is given by:

$$
\begin{aligned}
& A_{ \pm} \mathcal{J}_{j}^{m, q}(x)=\sqrt{(j \mp m)(j \pm m+1)} \mathcal{J}_{j}^{m \pm 1, q}(x), \\
& B_{ \pm} \mathcal{J}_{j}^{m, q}(x)=\sqrt{(j \mp q)(j \pm q+1)} \mathcal{J}_{j}^{m, q \pm 1}(x), \\
& C_{ \pm} \mathcal{J}_{j}^{m, q}(x)=\sqrt{\left(j+m+\frac{1}{2} \pm \frac{1}{2}\right)\left(j+q+\frac{1}{2} \pm \frac{1}{2}\right)} \mathcal{J}_{j \pm 1 / 2}^{m \pm 1 / 2, q \pm 1 / 2}(x), \\
& D_{ \pm} \mathcal{J}_{j}^{m, q}(x)=\sqrt{\left(j+m+\frac{1}{2} \pm \frac{1}{2}\right)\left(j-q+\frac{1}{2} \pm \frac{1}{2}\right)} \mathcal{J}_{j \pm 1 / 2}^{m \pm 1 / 2, q \mp 1 / 2}(x), \\
& E_{ \pm} \mathcal{J}_{j}^{m, q}(x)=\sqrt{\left(j-m+\frac{1}{2} \pm \frac{1}{2}\right)\left(j+q+\frac{1}{2} \pm \frac{1}{2}\right)} \mathcal{J}_{j \pm 1 / 2}^{m \mp 1 / 2, q \pm 1 / 2}(x), \\
& F_{ \pm} \mathcal{J}_{j}^{m, q}(x)=\sqrt{\left(j-m+\frac{1}{2} \pm \frac{1}{2}\right)\left(j-q+\frac{1}{2} \pm \frac{1}{2}\right)} \mathcal{J}_{j \pm 1 / 2}^{m \mp 1 / 2, q \mp 1 / 2}(x) .
\end{aligned}
$$

The three diagonal operators $J, M$ and $Q$

$$
(J, M, Q) \mathcal{J}_{j}^{m, q}(x)=(j, m, q) \mathcal{J}_{j}^{m, q}(x),
$$

belong to the Cartan subalgebra.

The 15-dimensional (conformal) simple Lie algebra $s u(2,2)$ has many applications in physics (see, for instance, [44 46] and references therein) and is a non-compact real form of the complex algebra $A_{3}$ [47, 48].

Note that the operators $A_{ \pm}\left(B_{ \pm}\right)$change the label $m(q)$ by \pm 1 leaving invariant the remaining two labels. They generate the representation $D_{j} \otimes D_{j}$ of the maximal compact subalgebra $s u(2) \oplus s u(2)$ of $s u(2,2)$. However, the operators $C_{ \pm}, \cdots, F_{ \pm}$ change all the $(j, m, q)$ by a half-integer quantity. Integer and half-integer values of $(j, m, q)$ are indeed related to a non-unitary and non-irreducible unique representation of $S U(2,2)$. Obviously the algebra is consistent with relation (5).

Furthermore composing the action of two pairs of operators (11) we can construct two operators that change $(j, m, q)$ to $(j \pm 1, m, q)$. Indeed $F_{ \pm} C_{ \pm}$ (equivalently, $C_{ \pm} F_{ \pm}, D_{ \pm} E_{ \pm}$or $E_{ \pm} D_{ \pm}$) are such operators. These new operators are second order differential operators but they can be reduced by means of the Jacobi equation (6) to first order differential operators when applied to the $\mathcal{J}_{j}^{m, q}[3]$. To define these new algebraic operators, we have to consider separately specific values of $m$ and $q$ : $j \geq|m|>|q|, j \geq|q|>|m|$ and $j \geq|m|=|q|$ :

1) For the states such that $j \geq|m|>|q|$ we can define from (11) and (12) two conjugate Hermitian operators as

$$
K_{ \pm}:=F_{ \pm} C_{ \pm} \frac{1}{\sqrt{(J+1 / 2 \pm 1 / 2)^{2}-Q^{2}}}
$$

for which the action on the $\mathcal{J}_{j}^{m, q}(x)$ does not depend from the value of $q$ :

$$
K_{ \pm} \mathcal{J}_{j}^{m, q}(x)=\sqrt{(j+1 / 2 \pm 1 / 2)^{2}-m^{2}} \mathcal{J}_{j \pm 1}^{m, q}(x) .
$$

Both operators (13) together with $K_{3}:=J+1 / 2$ close a $s u(1,1)$ Lie algebra

$$
\left[K_{+}, K_{-}\right]=-2 K_{3}, \quad\left[K_{3}, K_{ \pm}\right]= \pm K_{ \pm},
$$

i.e., the set $\left\{\mathcal{J}_{j}^{m, q}\right\}_{j \geq|m|>|q|}^{m, q \text { fixed }}$, with $|m|>|q|$, is a basis of the UIR of $S U(1,1)$ with Casimir $\mathcal{C}=$ $m^{2}-1 / 4$.

2) For the $\mathcal{J}_{j}^{m, q}(x)$ with $j \geq|q|>|m|$, the procedure is analogous. In fact, we only have to interchange $M \Longleftrightarrow Q$ in the definition of $K_{ \pm}$.

3) The third case shows a difficulty for the definition of the operator $K_{-}$as obtained from [3, 29] when $j=|m|=|q|$, since $K_{-}$is not well defined in eq.(13). The following limit allows to avoid this difficulty: 


$$
K_{-}:=\lim _{\epsilon \rightarrow 0}\left[\left(\left(1-X^{2}\right) D_{X}+X J+\frac{(M+\epsilon)(Q+\epsilon)}{J}\right) \frac{J}{\sqrt{J^{2}-(Q+\epsilon)^{2}}}\right],
$$

where $X$ and $D_{X}$ are the multiplication and derivative operators (i.e., $X f(x)=x f(x)$, $\left.D_{X} f(x)=d f(x) / d x\right)$. In Appendix B we study the continuity of the multiplication operator defined in $[3,29]$.

This limit removes the problem because the action of $K_{-}$on $\mathcal{J}_{j}^{m, q}(x)$ agrees with (14) for $j>|m|=$ $|q|$, while for $j=|m|=|q|$ vanishes because (16) is the product of two terms, one goes like $\epsilon$ and the other like $\epsilon^{-1 / 2}$. In conclusion, if $|m| \geq|q|$ the action of the $K_{ \pm}$is given by eqs. (14) with $j=|m|,|m|+1,|m|+2 \ldots$ and Casimir invariant $\mathcal{C}=m^{2}-1 / 4$, while for $|m|<|q|$, the result is the same after the exchange of $q$ and $m$. Thus, the set $\left\{\mathbb{J}_{j}^{m, q}\right\}_{j \geq \sup (|m|,|q|)}^{m, q \text { fixed }}$ supports, because of (10), a UIR of $S U(1,1)$ for any $m, q$.

All these operators are continuous linear mappings between suitable locally convex spaces admitting continuous extensions to dual spaces. The construction of these spaces as well as the discussion on their properties is the subject of the next sec-

$$
\left[p_{r, s}^{m, q}(f)\right]^{2}:=\sum_{j \geq \sup (|m|,|q|)}^{\infty}\left|f_{j}^{m, q}\right|^{2}(j+|m|+1)^{2 r}(j+|q|+1)^{2 s}<\infty .
$$

tion.

\section{BASIC SPACES AND THEIR OPERATORS}

\section{A. The spaces $\Phi_{m, q}$}

In the previous section, we have mentioned that for fixed $m$ and $q$ (both integer or halfinteger) the set of normalized Jacobi functions (9) $\left\{\mathrm{J}_{j}^{m, q}(x)\right\}_{j \geq \sup (|m|,|q|)}^{\infty}$ is an orthonormal basis for $L^{2}[-1,1]$. Let us construct a set of spaces $\Phi_{m, q}$ each depending on the labels $m$ and $q$, as follows: Let us take $f(x) \in L^{2}[-1,1]$, which admits the following expansion:

$$
f(x)=\sum_{j \geq \sup (|m|,|q|)}^{\infty} f_{j}^{m, q} \mathbb{J}_{j}^{m, q}(x) .
$$

Then by definition, $f(x) \in \Phi_{m, q}$ if and only if for all $r, s=0,1,2, \ldots$, we have that
Observe that the mappings $p_{r, s}^{m, q}(f)$ define a family of norms on $\Phi_{m, q}$ and hence a topology on each of the spaces $\Phi_{m, q}$. With this topology, $\Phi_{m, q}$ is a nuclear Frèchet space [49]. Each of the spaces $\Phi_{m, q}$ contains all the functions in the corresponding orthonormal basis, so that these spaces are all dense in $L^{2}[-1,1]$. Since the norm on $\Phi_{m, q}$ given by $r=s=0$ is just the norm on $L^{2}[-1,1]$, the topology in $\Phi_{m, q}$ is finer than the topology inherited from $L^{2}[-1,1]$, so that the canonical mapping $\Phi_{m, q} \longmapsto L^{2}[-1,1]$ is continuous. If we add the anti-dual $\Phi_{m, q}^{\times}$, we have for each of the values of $m$ and $q$ the RHS

$$
\Phi_{m, q} \subset L^{2}[-1,1] \subset \Phi_{m, q}^{\times} .
$$

Let $\mathcal{O}$ be a densely defined linear operator on
$L^{2}[-1,1]$ such that $\Phi_{m, q}$ reduces $\mathcal{O}$. This means that $\mathcal{O} \phi \in \Phi_{m, q}$ for all $\phi \in \Phi_{m, q}$, or equivalently that $\mathcal{O} \Phi_{m, q} \subset \Phi_{m, q}$, so that $\mathcal{O}: \Phi_{m, q} \longmapsto \Phi_{m, q}$. The operator $\mathcal{O}$ is continuous with respect to the locally convex topology induced by the family of norms $\left\{p_{r, s}^{m, q}\right\}_{r, s \in \mathbb{N}}^{m, q \text { fixed }}$, if and only if for each pair $(r, s)$, with $r, s=0,1,2, \ldots$, there exists a positive constant $K>0$ and a finite number of pairs $\left(r_{1}, s_{1}\right),\left(r_{2}, s_{2}\right), \ldots,\left(r_{k}, s_{k}\right)$, which depend on $(r, s)$, such that for all $f \in \Phi_{m, q}$, we have [50]

$p_{r, s}(\mathcal{O} f) \leq K\left\{p_{r_{1}, s_{1}}(f)+p_{r_{2}, s_{2}}(f)+\cdots+p_{r_{k}, s_{k}}(f)\right\}$,

where we have omitted the superindices $m$ and $q$ for simplicity. Along the present section, we shall omit these two indices unless necessary.

A similar formula proves the continuity of linear mappings between different locally convex spaces. 


\section{B. Operators acting on $\Phi_{m, q}$}

Our first goal is to prove that the operators $J, M$ and $Q$ defined in (12) are reduced by all the spaces $\Phi_{m, q}$ and are continuous as linear mappings on these spaces. Let us prove this property for $J$ first. After (9) and (12) it is clear that, for any $f(x) \in \Phi_{m, q}$ (17), we should define $J f$ as

$$
J f(x) \equiv J \sum_{j} f_{j} \mathbb{J}_{j}^{m, q}(x):=\sum_{j} f_{j} j \mathbb{J}_{j}^{m, q}(x) .
$$

Since

$$
\begin{aligned}
{\left[p_{r, s}(J f)\right]^{2} } & =\sum_{j}\left|f_{j}\right|^{2} j^{2}(j+|m|+1)^{2 r}(j+|q|+1)^{2 s} \\
& \leq \sum_{j}\left|f_{j}\right|^{2}(j+|m|+1)^{2(r+1)}(j+|q|+1)^{2 s}<\infty,
\end{aligned}
$$

(for all $r, s=0,1,2, \ldots$ ), we get that $(J f)(x) \in \Phi_{m, q}$ for any $f(x) \in \Phi_{m, q}$. In addition,

$$
\left[p_{r, s}(J f)\right]^{2} \leq \sum_{j}(j+|m|+1)^{2 r+2}(j+|q|+1)^{2 s}\left|f_{j}\right|^{2}=p_{r+1, s}^{2}(f),
$$

which proves the continuity of $J$. On $\Phi_{m, q}$ the operators $M$ and $Q$ are trivial, since they just multiply by a constant, either $m$ or $q$, respectively. Then, these operators are trivially continuous on all $\Phi_{m, q}$. The operators $K_{ \pm}$(14) are also linear and continuous on the spaces $\Phi_{m, q}$. Let us write $\left(K_{+} f\right)(x)$ for any $f(x) \in \Phi_{m, q}$ by using the expansion of $f(x)$ in the basis of the $\mathbb{J}_{j}^{m, q}(x)$ (17)

$$
\begin{aligned}
\left(K_{+} f\right)(x) & =\sum_{j} f_{j} K_{+} \mathbb{J}_{j}^{m, q}(x) \\
& =\sum_{j} f_{j} \sqrt{j+1 / 2} K_{+} \mathcal{J}_{j}^{m, q}(x) \\
& =\sum_{j} f_{j} \sqrt{(j+1)^{2}-m^{2}} \sqrt{j+1 / 2} \mathcal{J}_{j+1}^{m, q}(x) \\
& =\sum_{j} f_{j} \frac{\sqrt{(j+1)^{2}-m^{2}} \sqrt{j+1 / 2}}{\sqrt{j+3 / 2}} \mathbb{J}_{j+1}^{m, q}(x) .
\end{aligned}
$$

To show that $\left(K_{+} f\right)(x) \in \Phi_{m, q}$, we have to check that for any $r, s=0,1,2, \ldots$, the following series converge:

$$
\left[p_{r, s}\left(K_{+} f\right)\right]^{2}=\sum_{j}\left|f_{j}\right|^{2} \frac{j+1 / 2}{j+3 / 2}\left[(j+1)^{2}-m^{2}\right](j+1+|m|+1)^{2 r}(j+1+|q|+1)^{2 s} .
$$

Note that

$$
(j+|m|+2)^{2}=(j+|m|+1+1)^{2}=(j+|m|+1)^{2}+2(j+|m|+1)+1 \leq 4(j+|m|+1)^{2},
$$

where we have taken into account that if $a$ and $b$ are two positive numbers, then, $2 a b \leq a^{2}+b^{2}$. Also, we have that

$$
0 \leq(j+1)^{2}-m^{2} \leq(j+1)^{2}+m^{2} \leq(j+1)^{2}+m^{2}+2|m|(j+1)=(j+|m|+1)^{2},
$$

and $(j+1 / 2) /(j+3 / 2)<1$. If we use (24) and (25) in (23), we conclude that

$$
\left[p_{r, s}\left(K_{+} f\right)\right]^{2} \leq 2^{2 r+2 s} \sum_{j}\left|f_{j}\right|^{2}(j+|m|+1)^{2(r+1)}(j+|q|+1)^{2 s}=2^{2 r+2 s}\left[p_{r+1, s}(f)\right]^{2} .
$$

This proves that $K_{+} f \in \Phi_{m, q}$. In addition, the inequality (26) shows the continuity of $K_{+}$on $\Phi_{m, q}$, after (19). A similar proof works for $K_{-}$. Thus, we have concluded that $J, M, Q$ and $K_{ \pm}$are continuous linear operators on all the spaces $\Phi_{m, q}$. Furthermore, since $J, M, Q$ are symmetric on $\Phi_{m, q}$, they can be continuously extended into the duals $\Phi_{m, q}^{\times}$by using the duality formula (11). Same for $K_{ \pm}$as they are the formal adjoint of each other, i.e., $\left(K_{ \pm}\right)^{\dagger}=K_{\mp}$. 


\section{Operators mapping into different spaces $\Phi_{m, q}$}

Let us go back to equations (11) which define mappings between different $\Phi_{m, q}$. For instance, mappings $A_{ \pm}$and $B_{ \pm}$transform spaces with labels $m$ and $q$ integers (half-integers) into other with $m$ and $q$ also integers (half-integers). On the other hand, the remaining operators of (11) define mappings from integer (half-integer) indices into half-integer (integer) indices. In any case, these operators are well defined and continuous between their corresponding spaces. It is enough to prove this fact with $A_{+}$, being the proofs for the remaining cases similar. As usual, we consider the action of $A_{+}$on $f(x) \in \Phi_{m, q}$ as

$$
\begin{aligned}
\left(A_{+} f\right)(x) & =A_{+}\left(\sum_{j \geq \sup (|m|,|q|)} f_{j} \mathbb{J}_{j}^{m, q}(x)\right)=\sum_{j \geq \sup (|m|,|q|)} f_{j} A_{+} \mathbb{J}_{j}^{m, q}(x) \\
& =\sum_{j \geq \sup (|m+1|,|q|)} f_{j} \sqrt{(j-m)(j+m+1)} \mathbb{J}_{j}^{m+1, q}(x) .
\end{aligned}
$$

Observe that, if $|q|>|m|$, the same coefficients $f_{j}$ must appear in both sums in (27). On the contrary, if $|m| \geq|q|$, the term with coefficient $f_{j}$ with $j=|m|$, does not appear in the second sum in (27) since $A_{+} \mathcal{J}_{j}^{j, q}(x)=0$ (see (11) $)$. Next, let us consider the following series for any $r, s=0,1,2, \ldots$

$$
\left[p_{r, s}^{m+1, q}\left(A_{+} f\right)\right]^{2}=\sum_{j \geq \sup \{|m+1|,|q|\}}\left|f_{j}\right|^{2}[(j-m)(j+m+1)](j+|m+1|+1)^{2 r}(j+|q|+1)^{2 s},
$$

where $\left\{p_{r, s}^{m+1, q}\right\}_{r, s \in \mathbb{N}}$ is the set of seminorms in $\Phi_{m+1, q}$. Since,

$$
(j+|m+1|+1)^{2} \leq(j+|m|+1+1)^{2} \leq 4(j+|m|+1)^{2},
$$

we have that

$$
\left[p_{r, s}^{m+1, q}\left(A_{+} f\right)\right]^{2} \leq 2^{2} \sum_{j \geq \sup (|m|,|q|)}\left|f_{j}\right|^{2}(j+|m|+1)^{2(r+1)}(j+|q|+1)^{2 s}=\left[2 p_{r+1, s}^{m, q}(f)\right]^{2} .
$$

Then after (19), the linear mapping $A_{+}: \Phi_{m, q} \longmapsto \Phi_{m+1, q}$ is continuous. By a simple generalization of the duality formula (10), its adjoint, $A_{+}^{\dagger}$, is a linear continuous mapping between the duals: $A_{+}^{\dagger}$ : $\Phi_{m+1, q}^{\times} \longmapsto \Phi_{m, q}^{\times}$. Since $\Phi_{m, q} \subset \Phi_{m, q}^{\times}$for all $m$ and $q$, it is legitimate to investigate the form of the restriction of $A_{+}^{\dagger}$ to $\Phi_{m+1, q}$.

The duality formula gives for all the NAJF :

$$
\left\langle\mathbb{J}_{j^{\prime}}^{m+1, q} \mid A_{+} \mathbb{J}_{j}^{m, q}\right\rangle=\left\langle A_{+}^{\dagger} \mathbb{J}_{j^{\prime}}^{m+1, q} \mid \mathbb{J}_{j}^{m, q}\right\rangle,
$$

that gives the identity between two brackets. The l.h.s. of each bracket represents a functional acting on the vector in the r.h.s. of the bracket. Thus, $\mathbb{J}_{j^{\prime}}^{m+1, q}$ is a functional acting on the vector $A_{+} \mathbb{J}_{j}^{m, q} \in \Phi_{m+1, q}$ and $A_{+}^{\dagger} \mathbb{J}_{j^{\prime}}^{m+1, q}$ is a functional on $\mathbb{J}_{j}^{m, q} \in \Phi_{m, q}$. By elementary properties of RHS, this action coincides with the scalar product on the Hilbert space, which in the present case is $L^{2}[-1,1]$. This fact shows that

$$
\begin{aligned}
\left\langle\mathbb{J}_{j^{\prime}}^{m+1, q} \mid A_{+} \mathbb{J}_{j}^{m, q}\right\rangle & =\sqrt{(j-m)(j+m+1)}\left\langle\mathbb{J}_{j^{\prime}}^{m+1, q} \mid \mathbb{J}_{j}^{m+1, q}\right\rangle \\
& =\sqrt{(j-m)(j+m+1)} \delta_{j, j^{\prime}} \\
& =\left\langle\sqrt{(j-m)(j+m+1)} \mathbb{J}_{j}^{m, q} \mid \mathbb{J}_{j^{\prime}}^{m, q}\right\rangle=\left\langle A_{-} \mathbb{J}_{j}^{m+1, q} \mid \mathbb{J}_{j^{\prime}}^{m, q}\right\rangle,
\end{aligned}
$$

where the last identity in (29) comes from the ac-

tion of the operator $A_{-}$on the $\mathcal{J}_{j}^{m, q}$ after (11), 
i.e.

$$
A_{-} \mathbb{J}_{j}^{m+1, q}=\sqrt{(j+m+1)(j-m)} \mathbb{J}_{j}^{m, q} .
$$

Therefore, $A_{-}=A_{+}^{\dagger}$ on $\Phi_{m+1, q}$. Since $\Phi_{m+1, q}$ is dense in its dual $\Phi_{m+1, q}^{\times}$and the properties of RHS, $A_{-}$can be uniquely extended to a continuous linear mapping from $\Phi_{m+1, q}^{\times}$to $\Phi_{m, q}^{\times}$. This extension coincides with $A_{+}^{\dagger}$.

A similar discussion may be applied to all other mappings (11). In each case, it is obvious which are the involved spaces and which topologies we should endow on each of these spaces.

\section{BASES WITH $m$ AND $q$ FIXED}

So far, we have considered realizations of RHS of the form

$\Phi_{m, q} \subset L^{2}[-1,1] \subset \Phi_{m, q}^{\times}, \quad \forall m, q \in \mathbb{Z}($ or $\mathbb{Z}+1 / 2)$

where the elements of the test spaces $\Phi_{m, q}$ are functions.

We also may consider an abstract RHS $\Psi_{m, q} \subset$ $\mathcal{H}_{m, q} \subset \Psi_{m, q}^{\times}$, where the elements of each of the spaces are just vectors that we obtain by means of an unitary operator

$$
U: \mathcal{H}_{m, q} \longmapsto L^{2}[-1,1],
$$

such that $U \Psi_{m, q}=\Phi_{m, q}$ and $U$ is one-to-one and onto topology preserving mapping. Then, $U^{\dagger} \Phi_{m, q}=U^{-1} \Phi_{m, q}=\Psi_{m, q}$ has the same properties. With the aid of the duality formula similar to (11),

$\left\langle F \mid U^{\dagger} \varphi\right\rangle=\langle U F \mid \varphi\rangle, \quad \forall \varphi \in \Psi_{m, q}, \forall F \in \Psi_{m, q}^{\times}$,

we may extend $U$ to a linear continuous one-to-one mapping $U \Psi_{m, q}^{\times}=\Phi_{m, q}^{\times}$, preserving topologies. In other words, we have the following diagram

$$
\begin{array}{cccc}
\Psi_{m, q} & \subset & \mathcal{H}_{m, q} & \subset \Psi_{m, q}^{\times} \\
U \downarrow & & U \downarrow & U \downarrow \\
\Phi_{m, q} & \subset & L^{2}[-1,1] & \subset \Phi_{m, q}^{\times}
\end{array} .
$$

\section{A. Discrete basis}

For the forthcoming discussion, we need to introduce some new ingredients. These are some new RHS to be defined later. In any case, for each of the values of $m$ and $q$, we shall define in Section IVC a space of test functions $\Xi_{m, q}$ that include all the $\mathrm{NAJF} \mathbb{J}_{j}^{m, q}(x)$ with $j \geq \sup (|m|,|q|)$. The spaces $\Xi_{m, q}$ will be different from the spaces $\Phi_{m, q}$, although they will play a similar role. Then for fixed $m$ and $q$, we take $\Omega_{m, q}:=U^{-1} \Xi_{m, q}$, as well as $\widetilde{K}_{ \pm}:=U^{-1} K_{ \pm} U$ and

$$
|j,(m, q)\rangle:=U^{-1} \mathbb{J}_{j}^{m, q}=U^{-1} \sqrt{j+1 / 2} \mathcal{J}_{j}^{m, q}(x)=\sqrt{j+1 / 2} U^{-1} \mathcal{J}_{j}^{m, q}(x) .
$$

After (22), we have that

$$
\begin{aligned}
\widetilde{K}_{+}|j,(m, q)\rangle & =\frac{\sqrt{(j+1)^{2}-m^{2}} \sqrt{j+1 / 2}}{\sqrt{j+3 / 2}}|j+1,(m, q)\rangle, \\
\widetilde{K}_{-}|j,(m, q)\rangle & =\frac{\sqrt{j^{2}-m^{2}} \sqrt{j+1 / 2}}{\sqrt{j-1 / 2}}|j-1,(m, q)\rangle,
\end{aligned}
$$

with $\widetilde{K}_{-}|0,(0,0)\rangle=0$. For $m$ and $q$ fixed and $j \geq \sup \{|m|,|q|\}$, the vectors $|j,(m, q)\rangle$ form a basis in $\mathcal{H}$, so that

$$
\left\langle j,(m, q) \mid j^{\prime},(m, q)\right\rangle=\delta_{j, j^{\prime}}, \quad \sum_{j}|j,(m, q)\rangle\langle j,(m, q)|=\mathcal{I},
$$

where $\mathcal{I}$ is the identity on $\mathcal{H}_{m, q}$ and unless otherwise stated, sum on $j$ will run from $j \geq \sup \{|m|,|q|\}$ to infinite. 


\section{B. Continuous basis}

Next, we formally construct a continuous basis for $\Phi_{m, q}$ as follows: for any $x \in[-1,1]$, we define

$$
|x,(m, q)\rangle:=\sum_{j}|j,(m, q)\rangle \mathbb{J}_{j}^{m, q}(x)
$$

This formal definition, along standard manipulations give some properties for kets $|x,(m, q)\rangle$ such as [29]

$$
\left\langle x,(m, q) \mid x^{\prime},(m, q)\right\rangle=\delta\left(x-x^{\prime}\right), \quad \int_{-1}^{1}|x,(m, q)\rangle d x\langle x,(m, q)|=I,
$$

where $I$ is some sort of identity. In addition,

$$
|j,(m, q)\rangle=\int_{-1}^{1}|x,(m, q)\rangle \mathbb{J}_{j}^{m, q}(x) d x
$$

and

$$
\mathbb{J}_{j}^{m, q}(x)=\langle x,(m, q) \mid j,(m, q)\rangle=\langle j,(m, q) \mid x,(m, q)\rangle .
$$

Note that the $\left\{\mathbb{J}_{j}^{m, q}(x)\right\}$ are the (real) transition matrices between the discrete and continuous bases of elements $|j,(m, q)\rangle$ and $|x,(m, q)\rangle$.

For any arbitrary $|f\rangle \in \Phi_{m, q}$, we have two different expressions depending the basis where it is spanned

$$
f \equiv|f\rangle=\left\{\begin{array}{c}
\int_{-1}^{1} d x|x,(m, q)\rangle f^{m, q}(x) \\
\sum_{j \geq \sup (|m|,|q|)} f_{j}^{m, q}|j,(m, q)\rangle
\end{array}\right.
$$

with

$$
\begin{aligned}
f^{m, q}(x) & =\langle x,(m, q) \mid f\rangle=\sum_{j} f_{j}^{m, q} \mathbb{J}_{j}^{m, q}(x) \\
f_{j}^{m, q} & =\langle j,(m, q) \mid f\rangle=\int_{-1}^{1} \mathbb{J}_{j}^{m, q}(x) f^{m, q}(x) d x
\end{aligned}
$$

Thus, we have obtained a relation between discrete, $\{|j,(m, q)\rangle\}_{j \geq \sup (|m|,|q|)}$, and continuous basis, $\{|x,(m, q)\rangle\}_{x \in[-1,1]}$.

\section{The spaces of test functions $\Xi_{m, q}$}

The next goal is to provide a good definition of the kets $|x,(m, q)\rangle$. This is why we need a new RHS with spaces of test functions denoted by $\Xi_{m, q}$. These functions should have the following properties:

1. Each function $f(x) \in \Xi_{m, q}$ is of the form (17).

2. For any $r, s=0,1,2, \ldots$, we have that the coefficients in the series (17) satisfy

$$
t_{r, s}^{m, q}(f):=\sum_{j \geq \sup (|m|,|q|)}^{\infty}\left|a_{j}\right|(j+|m|+1)^{r}(j+|q|+1)^{s}<\infty .
$$


Relation (40) implies that the series (17) converge in the Hilbert space norm as well as pointwise and absolutely. For convenience in the presentation, we shall prove this properties later.

In the sequel, we shall denote the mappings $t_{r, s}^{m, q}$ as $t_{r, s}$ unless otherwise stated to avoid confusion. These, $t_{r, s}$ (for $r, s=0,1,2, \ldots$ ) are a family of norms on $\Xi_{m, q}$ that define the topology on $\Xi_{m, q}$. To complete a RHS, one has to add $L^{2}[-1,1]$ as Hilbert space. The spaces $\Xi_{m, q}$ are dense in $L^{2}[-1,1]$ since they contain all finite linear combinations of $\mathbb{J}_{j}^{m, q}(x)$. However, we need more information to conclude that $\Xi_{m, q} \subset L^{2}[-1,1] \subset \Xi_{m, q}^{\times}$are RHS. Here, we define $\Xi_{m, q}^{\times}$as usual and note that this dual space is the same no matter whether $\Xi_{m, q}$ is complete or not. This extra information is giving by showing that the canonical injection

$$
\begin{array}{rlr}
\mathfrak{j}: \Xi_{m, q} & \longmapsto & L^{2}[-1,1] \\
f & \longmapsto & j(f)
\end{array}
$$

is continuous. This proof is very simple: since

$$
\|j(f)\|=\sqrt{\sum_{j \geq \sup (|m|,|q|)}\left|f_{j}\right|^{2}} \leq \sum_{j \geq \sup (|m|,|q|)}\left|f_{j}\right|=t_{0,0}(f),
$$

where $\|-\|$ denotes the Hilbert norm on $L^{2}[-1,1]$. Then, the conclusion comes from a simple generalization of (19). Thus,

$$
\Xi_{m, q} \subset L^{2}[-1,1] \subset \Xi_{m, q}^{\times}
$$

is a RHS.

Now, combine (40) with $r=s=0$ along to the inequality in (41) to prove our assertion that the series in (17) satisfying (40) converges in the Hilbert space norm. Pointwise and absolute convergences will be a consequence of the Weisstrass M-theorem [51] as shall see later.

\section{The spaces of test vectors $\Omega_{m, q}$}

Let us consider the unitary operator $U$ introduced in (30) and define the spaces $\Omega_{m, q}:=U^{-1} \Xi_{m, q}$, for each pair of values $m, q$. Let us transport the topology defined on the space of test functions $\Xi_{m, q}$ to the space of test vectors $\Omega_{m, q}$ by means of $U^{-1}$. It comes that for any $|f\rangle=$ $\sum_{j} f_{j}^{m, q}|j,(m, q)\rangle \in \varnothing_{m, q}$, the norms defining the topology on $\varnothing_{m, q}$ are equal to $t_{r, s}(f)$ defined as in (40), see also the line below (40). For fixed $m$ and $q$, the triplet $\Xi_{m, q} \subset L^{2}[-1,1] \subset \Xi_{m, q}^{\times}$is a realization of the abstract RHS $\Omega_{m, q} \subset \mathcal{H} \subset \Omega_{m, q}^{\times}$by $U$. In other words,

$$
\begin{array}{cccc}
\emptyset_{m, q} & \subset & \mathcal{H} & \subset \varnothing_{m, q}^{\times} \\
U \downarrow & & U \downarrow & U \downarrow \\
\Xi_{m, q} & \subset & L^{2}[-1,1] & \subset \Xi_{m, q}^{\times}
\end{array} .
$$

Let us discuss the validity of formula (34) to define the kets $|x,(m, q)\rangle$. To do it, we have to take into account the following inequality valid for the Algebraic Jacobi Functions (see Appendix C):

$$
\left|\mathcal{J}_{j}^{m, q}(x)\right| \leq(j+|m|+1)(j+|q|+1), \quad \forall j \geq|m|,|q| ; 2 j \in \mathbb{N} ; j-m, j-q \in \mathbb{N},
$$

that for the NAJF (9) becomes

$$
\left|\mathbb{J}_{j}^{m, q}(x)\right| \leq \sqrt{j+1 / 2}(j+|m|+1)(j+|q|+1) .
$$

Next, let us consider $|f\rangle \in \emptyset_{m, q}$. From equations (34), (38), (39) and (9) we have that

$$
f^{m, q}(x):=\langle x,(m, q) \mid f\rangle=\sum_{j}\langle j,(m, q) \mid f\rangle \mathbb{J}_{j}^{m, q}(x),
$$

where from now on the sums in $j$ are always extended from $\sup (|m|,|q|)$ up to infinite, unless otherwise stated. 
Condition (40) implies the absolute and pointwise convergence of the series (17). Indeed, let us consider

$$
\begin{aligned}
\sum_{j}\left|f_{j}\right|\left|\mathbb{J}_{j}^{m, q}(x)\right| & \leq \sum_{j}\left|f_{j}\right| \sqrt{j+1 / 2}(j+|m|+1)(j+|q|+1) \\
& \leq \sum_{j}\left|f_{j}\right|(j+|m|+1)^{2}(j+|q|+1),
\end{aligned}
$$

which converges by condition (40). The first inequality in (45) is due to (43), while the second one is trivial. Then, the Weierstrass M-theorem shows our assertion.

Since the convergence is now pointwise after our hypothesis, we may take modulus in both sides of (44) and remembering (40) so as to obtain the following inequality:

$$
\begin{aligned}
|\langle f \mid x,(m, q)\rangle| & \leq \sum_{j}|\langle f \mid j,(m, q)\rangle| \cdot\left|\mathbb{J}_{j}^{m, q}(x)\right| \\
& \leq \sum_{j}\left|f_{j}\right| \sqrt{j+1 / 2}(j+|m|+1)(j+|q|+1) \\
& \leq \sum_{j}^{j}\left|f_{j}\right|(j+|m|+1)^{2}(j+|q|+1)=t_{2,1}(f) .
\end{aligned}
$$

The inequality (46) along the linearity of the functional $|x,(m, q)\rangle$ on $\Omega_{m, q}$ prove the continuity of $|x,(m, q)\rangle$ on $\Omega_{m, q}$, and hence that $|x,(m, q)\rangle \in \Omega_{m, q}^{\times}$. Note that, while $|f\rangle \in \varnothing_{m, q}$, its realization as function is given by $\langle f \mid x,(m, q)\rangle$, which is in $\Xi_{m, q}$.

Next, let us prove the continuity of $\widetilde{K}_{ \pm}$on $\emptyset_{m, q}$. For arbitrary $|f\rangle=\sum_{j} f_{j}|j,(m, q)\rangle \in \emptyset_{m, q}$, we shall prove in just one operation that $\widetilde{K}_{ \pm}|f\rangle \in \varnothing_{m, q}$ and that $\widetilde{K}_{ \pm}$are continuous on $\varnothing_{m, q}$. By definition, the action of $\widetilde{K}_{ \pm}$on $\varnothing_{m, q}$ is given by

$$
\widetilde{K}_{ \pm}|f\rangle:=\sum_{j} f_{j} \widetilde{K}_{ \pm}|j,(m, q)\rangle, \quad \forall|f\rangle \in \varnothing_{m, q} .
$$

After the action of $\widetilde{K}_{ \pm}$on the basis vectors given in (32), we have for $\widetilde{K}_{+}$that (see (18))

$$
t_{r, s}\left(K_{+}|f\rangle\right)=\sum_{j}\left|f_{j}\right| \sqrt{(j+1)^{2}-m^{2}}(j+1+|m|+1)^{r}(j+1+|q|+1)^{s},
$$

for any $r, s,=0,1,2, \ldots$ Then, and taking into account that

$$
(j+1)^{2}-m^{2} \leq(j+|m|+1)^{2} \quad \text { and } \quad(j+1+|m|+1) \leq 2(j+|m|+1),
$$

we get that

$$
t_{r, s}\left(K_{+}|f\rangle\right) \leq 2^{r+s} \sum_{j}\left|f_{j}\right|(j+|m|+1)^{r+2}(j+|q|+1)^{s+1}=2^{r+s} t_{r+2, s+1}(f),
$$

or, in other words,

$$
t_{r, s}\left(K_{+}|f\rangle\right) \leq 2^{r+s} t_{r+2, s+1}(f), \quad \forall|f\rangle \in \varnothing_{m, q} .
$$

This proves our claim for $\widetilde{K}_{+}$. A similar proof will show that $\widetilde{K}_{-}$satisfies the same properties. In particular, these operators can be continuously extended into the dual $\varnothing_{m, q}{ }^{\times}$. The same is true for $K_{ \pm}$ acting on $\Xi_{m, q}$.

It is worthy to notice that as a consequence of the fact that $\left(\sum\left|f_{n}\right|^{2}\right)^{1 / 2} \leq \sum\left|f_{n}\right|$ (see (41)) one has that

$$
p_{r, s}(f) \leq t_{r, s}(f), \quad \forall|f\rangle \in \varnothing_{m, q},
$$


where $p_{r, s}$ were defined in (28) and $t_{r, s}^{m, q}$ in (40). These properties have interesting consequences: first of all $\Xi_{m, q} \subset \Phi_{m, q}$ and second that the canonical injection $\mathfrak{j}: \Xi_{m, q} \longmapsto \Phi_{m, q}, \mathfrak{j}(f):=f$, is continuous. Thus, we have the relations:

$$
\Xi_{m, q} \subset \Phi_{m, q} \subset L^{2}[-1,1] \subset \Phi_{m, q}^{\times} \subset \Xi_{m, q}^{\times},
$$

where all canonical injections (identities) are continuous. Same properties are valid for the chain of abstract spaces:

$$
\emptyset_{m, q} \subset \Psi_{m, q} \subset \mathcal{H} \subset \Psi_{m, q}^{\times} \subset \varnothing_{m, q}^{\times}
$$

Thus, the topology given by the norms $t_{r, s}$ makes the linear functionals $|x,(m, q)\rangle$ on $\Omega_{m, q}$ continuous, a property that may not be true if instead we would have used the topology produced by the norms $p_{r, s}$ defined on each of the spaces $\Psi_{m, q}$.

We finish this section by adding that the operators $J, M$ and $Q$ as defined in (31) are also continuous on all the spaces $\Xi_{m, q}$. The proof is immediate. Also there are some relations between spaces which are also trivially continuous. For instance:

$$
\Xi_{m, q} \stackrel{j}{\longrightarrow} \Phi_{m, q} \stackrel{A_{+}}{\longrightarrow} \Phi^{m+1, q},
$$

where $j$ is the identity. Similar relations can be constructed with the operators defined in the list (11).

\section{JACOBI HARMONICS AND JACOBI FERMION HARMONICS}

The Associated Legendre polynomials (that depend on two parameters and one variable) are related to the Spherical Harmonics (two parameters and two variables). Similarly the Algebraic Jacobi Functions, that depend on three parameters and one variable, can be related to the "Jacobi Harmonics"

$$
\mathcal{N}_{j}^{m, q}(x, \phi, \chi):=\mathbb{J}_{j}^{m, q}(x) e^{i m \phi} e^{i q \chi}=\sqrt{j+1 / 2} \mathcal{J}_{j}^{m, q}(x) e^{i m \phi} e^{i q \chi},
$$

with three discrete parameters $(j, m, q)$ and three continuous variables $(x, \phi, \chi)$.

We have to distinguish two cases depending if $(j, m, q)$ are integer or half-integer. If they are integer the range of the angular variables is $\phi \in[0,2 \pi)$ and $\chi \in[0, \pi]$, and we call $\mathcal{N}_{j}^{m, q}(x, \phi, \chi)$ properly Jacobi Harmonics while if they are half-integer $\phi \in[0,4 \pi)$ and $\chi \in[0,2 \pi)$ and we call them "Jacobi Fermion Harmonics".

Indeed, the Jacobi Harmonics are a generalization of the Spherical Harmonics: assuming $x:=\cos \theta$ we have $\theta \in[0, \pi]$ and the Jacobi Harmonics are defined in the hypersphere $S^{3}$, and satisfy the relations of orthogonality and completeness

$$
\begin{aligned}
\frac{1}{2 \pi^{2}} \int_{0}^{2 \pi} d \phi \int_{0}^{\pi} d \chi \int_{-1}^{1} d x \mathcal{N}_{j}^{m, q}(x, \phi, \chi)\left(\mathcal{N}_{j^{\prime}}^{m^{\prime}, q^{\prime}}\right)^{*}(x, \phi, \chi) & =\delta_{j, j^{\prime}} \delta_{m, m^{\prime}} \delta_{q, q^{\prime}}, \\
\sum_{j=0}^{\infty} \sum_{m, q=-j}^{j}\left[\mathcal{N}_{j}^{m, q}(x, \phi, \chi)\right]^{*} \mathcal{N}_{j}^{m, q}\left(x^{\prime}, \phi^{\prime}, \chi^{\prime}\right) & =\delta\left(x-x^{\prime}\right) \delta\left(\phi-\phi^{\prime}\right) \delta\left(\chi-\chi^{\prime}\right) .
\end{aligned}
$$

The Jacobi Harmonics thus behave on $S^{3}$ like the Spherical Harmonics on $S^{2}$ [27]. On the other hand, for $j$ half-integer the Jacobi Fermion Harmonics are the generalization of the Fermionic quasi-Spherical Harmonics [52] because they verify

$$
\begin{aligned}
\frac{1}{8 \pi^{2}} \int_{0}^{4 \pi} d \phi \int_{0}^{2 \pi} d \chi \int_{-1}^{1} d x \mathcal{N}_{j}^{m, q}(x, \phi, \chi)\left(\mathcal{N}_{j^{\prime}}^{m^{\prime}, q^{\prime}}\right)^{*}(x, \phi, \chi) & =\delta_{j, j^{\prime}} \delta_{m, m^{\prime}} \delta_{q, q^{\prime}}, \\
\sum_{j=1 / 2}^{\infty} \sum_{m, q}\left[\mathcal{N}_{j}^{m, q}(x, \phi, \chi)\right]^{*} \mathcal{N}_{j}^{m, q}\left(x^{\prime}, \phi^{\prime}, \chi^{\prime}\right) & =\delta\left(x-x^{\prime}\right) \delta\left(\phi-\phi^{\prime}\right) \delta\left(\chi-\chi^{\prime}\right) .
\end{aligned}
$$


For any fixed $j$ no matter whether integer or half-integer, the functions $e^{i m \phi}$ and $e^{i q \chi}$ span respective vector spaces of dimension $2 j+1$, which are isomorphic to $\mathbb{C}^{2 j+1}$. Therefore, we may identify these spaces of functions with $\mathbb{C}^{2 j+1}$, and establish the following result:

Theorem.- The set of functions $\mathcal{N}_{j}^{m, q}(x, \phi, \chi)$ with $m, q=-j,-j+1, \ldots, j-1, j$ and $j=0,1,2, \ldots$, or $j=1 / 2,3 / 2 \ldots$, is a basis of the Hilbert spaces:

$$
\mathcal{L}_{H}=\bigoplus_{j=0}^{\infty} L^{2}[-1,1] \otimes \mathbb{C}^{2 j+1} \otimes \mathbb{C}^{2 j+1}, \quad \mathcal{L}_{F}:=\bigoplus_{j=1 / 2}^{\infty} L^{2}[-1,1] \otimes \mathbb{C}^{2 j+1} \otimes \mathbb{C}^{2 j+1} .
$$

The subindices $H$ and F stand for "Jacobi Harmonic" and "Jacobi Fermion Harmonic", respectively.

Proof. It is a direct outcome of (48) and (49).

As a consequence, functions $f_{H}(x, \phi, \chi) \in \mathcal{L}_{H}$ and in $f_{F}(x, \phi, \chi) \in \mathcal{L}_{F}$ admit the following expansions, which converge in the Hilbert norm:

$$
\begin{aligned}
& f_{H}(x, \phi, \chi)=\sum_{j=0}^{\infty} \sum_{m, q=-j}^{j} f_{m, q}^{(H) j} \mathcal{N}_{j}^{m, q}(x, \phi, \chi), \quad\left\|f_{H}(x, \phi, \chi)\right\|^{2}=\sum_{j, m, q}\left|f_{m, q}^{(H) j}\right|^{2}<\infty, \\
& f_{F}(x, \phi, \chi)=\sum_{j=1 / 2}^{\infty} \sum_{m, q=-j}^{j} f_{m, q}^{(F) j} \mathcal{N}_{j}^{m, q}(x, \phi, \chi), \quad\left\|f_{F}(x, \phi, \chi)\right\|^{2}=\sum_{j, m, q}\left|f_{m, q}^{(F) j}\right|^{2}<\infty .
\end{aligned}
$$

Let $\Phi_{H} \subset \mathcal{L}_{H}$ and $\Phi_{F} \subset \mathcal{L}_{F}$ be the spaces of functions $f_{H}(x, \phi, \chi)$ and $f_{F}(x, \phi, \chi)$ as in (50) and (51), respectively, that for all $r, s=0,1,2, \ldots$, verify

$$
\begin{aligned}
{\left[p_{r, s}^{H}\left(f_{H}\right)\right]^{2} } & :=\sum_{j=0}^{\infty} \sum_{m, q=-j}^{j}\left|f_{m, q}^{(H) j}\right|^{2}(j+|m|+1)^{2 r}(j+|q|+1)^{2 s}<\infty, \\
{\left[p_{r, s}^{F}\left(f_{F}\right)\right]^{2} } & :=\sum_{j=1 / 2}^{\infty} \sum_{m, q=-j}^{j}\left|f_{m, q}^{(F) j}\right|^{2}(j+|m|+1)^{2 r}(j+|q|+1)^{2 s}<\infty .
\end{aligned}
$$

For each $r, s=0,1,2, \ldots, p_{r, s}^{H, F}\left(f_{H, F}\right)$ are norms for the function $f_{H, F}$. With the topology produced by these norms, $\Phi_{H}$ and $\Phi_{F}$ are Fréchet nuclear spaces. In addition, $\Phi_{H}$ and $\Phi_{F}$ contain the functions in the respective basis of $\mathcal{H}_{H}$ and $\mathcal{H}_{F}$, so that they are dense in the corresponding Hilbert space. Furthermore, for $r=s=0$, we obtain the Hilbert norm, so that the canonical injections $\mathfrak{j}_{H, F}: \Phi_{H, F} \longmapsto \mathcal{L}_{H, F}$ are continuous. All these facts imply that

$$
\Phi_{H, F} \subset \mathcal{L}_{H, F} \subset \Phi_{H, F}^{\times}
$$

and also

$$
\Phi_{H \oplus F} \subset \mathcal{L}_{H \oplus F} \subset \Phi_{H \oplus F}^{\times},
$$

where $\Phi_{H \oplus F}=\Phi_{H} \oplus \Phi_{F}$ and $\mathcal{L}_{H \oplus F}=\mathcal{L}_{H} \oplus \mathcal{L}_{F}$, are all rigged Hilbert spaces.

\section{A. Operators acting on $\mathcal{L}_{H \oplus F}$}

The set of Jacobi functions $\left\{\mathcal{N}_{j}^{m, q}(x, \phi, \chi)\right\}_{j, m, q \in N / 2}$ supports a representation of $S U(2,2)$, that contrarily to what happens for the $\mathbb{J}_{j}^{m, q}(x)$, is also unitary and irreducible. Its generators are related with those 
of (11) by

$$
\begin{array}{ll}
\mathcal{A}_{ \pm}=A_{ \pm} e^{ \pm i \phi}, & \mathcal{B}_{ \pm}=B_{ \pm} e^{ \pm i \chi}, \\
\mathcal{C}_{ \pm}=C_{ \pm} e^{ \pm i \phi / 2} e^{ \pm i \chi / 2}, & \mathcal{D}_{ \pm}=D_{ \pm} e^{ \pm i \phi / 2} e^{\mp i \chi / 2}, \\
\mathcal{E}_{ \pm}=E_{ \pm} e^{\mp i \phi / 2} e^{ \pm i \chi / 2}, & \mathcal{F}_{ \pm}=F_{ \pm} e^{\mp i \phi / 2} e^{\mp i \chi / 2},
\end{array}
$$

while operators (12) remain invariant, i.e, $\mathcal{J}=J, \mathcal{M}=M, \mathcal{Q}=Q$. Moreover, for $(m, q)$ fixed the functions $\mathcal{N}_{j}^{m, q}(x, \phi, \chi)$ support a UIR of $S U(1,1)$ generated by the same operators (15), i.e., $\mathcal{K}_{ \pm}=$ $K_{ \pm}, \mathcal{K}_{3}=K_{3}$.

All the above mentioned operators are continuous as we prove at the following. The proof of the continuity of $\mathcal{J}$ on $\Phi_{H}$ (and on $\Phi_{F}$ ) is similar to that given in (20) and (21) for $J$. For $\mathcal{M}$ on $\Phi_{H}$ we have

$$
\left(\mathcal{M} f_{H}\right)(x, \phi, \chi):=\sum_{j=0}^{\infty} \sum_{m, q} m f_{m, q}^{(H) j} \mathcal{N}_{j}^{m, q}(x, \phi, \chi), \quad \forall f_{H}(x, \phi, \chi) \in \Phi_{H}
$$

Then,

$$
\begin{aligned}
{\left[p_{r, s}^{H}\left(\mathcal{M} f_{H}\right)\right]^{2} } & =\sum_{j=0}^{\infty} \sum_{m, q}\left|f_{m, q}^{(H) j}\right|^{2} m^{2}(j+|m|+1)^{2 r}(j+|q|+1)^{2 s} \\
& \leq \sum_{j=0}^{\infty} \sum_{m, q}\left|f_{m, q}^{(H) j}\right|^{2}(j+|m|+1)^{2(r+1)}(j+|q|+1)^{2 s}=\left[p_{r+1, s}^{H}\left(f_{H}\right)\right]^{2},
\end{aligned}
$$

which proves that $\mathcal{M} \Phi_{H} \subset \Phi_{H}$ with continuity. Same proof is similar for $\mathcal{M}$ on $\Phi_{F}$ and for $\mathcal{Q}$ on both spaces. A consequence is that we can extend $\mathcal{J}, \mathcal{M}$ and $\mathcal{Q}$ to the duals $\left(\Phi_{H, F}\right)^{\times}$with continuity with respect to the weak topology.

The operators $\mathcal{A}_{ \pm}$are reduced by the spaces $\Phi_{H, F}$ (i.e., $\mathcal{A}_{ \pm} \Phi_{H, F} \subset \Phi_{H, F}$ ) as well as $\mathcal{B}_{ \pm}$and $\mathcal{K}_{ \pm}$. For $\mathcal{A}_{+}$on $\Phi_{H}$ we should define the action of $\mathcal{A}_{+}$as

$$
\left(\mathcal{A}_{+} f_{H}\right)(x, \phi, \chi):=\sum_{j=0}^{\infty} \sum_{m, q} f_{m, q}^{(H) j} \sqrt{(j-m)(j+m+1)} \mathcal{N}_{j}^{m+1, q}(x, \phi, \chi) .
$$

To prove that $\left(\mathcal{A}_{+} f_{H}\right)(x, \phi, \chi)$ is in $\Phi_{H}$, we have to show that the series

$$
\sum_{j=0}^{\infty} \sum_{m, q}\left|f_{m, q}^{(H) j}\right|^{2}|(j-m)(j+m+1)|(j+|m+1|+1)^{2 r}(j+|q|+1)^{2 s}
$$

converges for $r, s=0,1,2, \ldots$ Clearly, $j+|m+1|+1 \leq 2(j+|m|+1)$, so that (53) is smaller or equal to

$$
2^{2 r} \sum_{j, m, q}\left|f_{m, q}^{(H) j}\right|^{2}(j+|m|+1)^{2(r+1)}(j+|q|+1)^{2 s}
$$

which converges for all $r, s=0,1,2, \ldots$ This shows that, for all $f_{H}(x, \phi, \chi) \in \Phi_{H}$, we have that $\left(\mathcal{A}_{+} f_{H}\right)(x, \phi, \chi) \in \Phi_{H}$. Moreover, this discussion also concludes that

$$
\left[p_{r, s}^{H}\left(\mathcal{A}_{+} f_{H}\right)\right]^{2} \leq 2^{2 r}\left[p_{r+1, s}^{H}\left(f_{H}\right)\right]^{2}, \quad \forall f_{H} \in \Phi_{H},
$$

which, after (19), shows the continuity of $\mathcal{A}_{+}$on $\Phi_{H}$. A similar argument is valid on $\Phi_{F}$ and the same considerations are in order for $\mathcal{A}_{-}, \mathcal{B}_{ \pm}$and $\mathcal{K}_{ \pm}$on $\Phi_{H}$ and $\Phi_{F}$.

Also the discussion at the end Subsection IIIC referred to the adjoint operator $A_{+}^{\dagger}$ can be repeated now for $\Phi_{H}$ and $\Phi_{F}$. Thus, $\mathcal{A}_{+}^{\dagger}=\mathcal{A}_{-}$can be weakly extended to a continuous linear mapping on $\left(\Phi_{H, F}\right)^{\times}$ such that $\mathcal{A}_{+}^{\dagger} \Phi_{H, F}^{\times} \subset \Phi_{H, F}^{\times}$, and analogously for $\mathcal{A}_{-}^{\dagger}, \mathcal{B}_{ \pm}^{\dagger}, \mathcal{K}_{ \pm}^{\dagger}$. 
The remaining operators of (52) $\left(\mathcal{C}_{ \pm}, \mathcal{D}_{ \pm}, \mathcal{E}_{ \pm}, \mathcal{F}_{ \pm}\right)$have a slight different nature. In fact, they transform Jacobi Harmonics into Jacobi Fermion Harmonics and vice-versa, as may be seen from (11) and (52). As a simple consequence, these operators are mappings from $\Phi_{H}$ into $\Phi_{F}$ and vice-versa. The proof of the continuity of all these operators on these spaces can be made by similar arguments to those used so far for $\mathcal{A}_{+}$.

Moreover they are formal adjoint of each other, i.e., for $\mathcal{C}_{+}$and $\mathcal{C}_{-}$

$$
\left\langle\mathcal{C}_{ \pm} f_{H} \mid g_{F}\right\rangle=\left\langle f_{H} \mid \mathcal{C}_{\mp} g_{F}\right\rangle, \quad \forall f_{H} \in \Phi_{H}, \quad \forall g_{F} \in \Phi_{F} .
$$

If $g_{F} \in \Phi_{F}^{\times}$, the dual space of $\Phi_{F}$, the duality formula $\left\langle\mathcal{C}_{ \pm} f_{H} \mid g_{F}\right\rangle=\left\langle f_{H} \mid \mathcal{C}_{ \pm}^{\dagger} g_{F}\right\rangle$, defines a weakly continuous mapping $\mathcal{C}_{ \pm}^{\dagger}$ from $\Phi_{F}^{\times}$to $\Phi_{H}^{\times}$. This mapping obviously extends $\mathcal{C}_{\mp}$. The same is true if we interchange the role between the spaces. Resuming, we have

$$
\begin{array}{lll}
\mathcal{C}_{ \pm}^{\dagger}: \Phi_{H}^{\times} \longmapsto \Phi_{F}^{\times} & \text {extend } \quad \mathcal{C}_{\mp}: \Phi_{F} \longmapsto \Phi_{H}, \\
\mathcal{C}_{ \pm}^{\dagger}: \Phi_{F}^{\times} \longmapsto \Phi_{H}^{\times} & \text {extend } \quad \mathcal{C}_{\mp}: \Phi_{H} \longmapsto \Phi_{F} .
\end{array}
$$

Similar relations hold for the other pairs $\mathcal{D}_{ \pm}, \mathcal{E}_{ \pm}$and $\mathcal{F}_{ \pm}$.

These results obtained for the generators of the Lie algebra $s u(2,2)$ can be extended to its Universal Enveloping Algebra, UEA $[s u(2,2)]$, in the sense that all elements of $\mathrm{UEA}[s u(2,2)]$ are continuous on $\Phi_{H \oplus F}$ and can be continuously extended to weakly continuous mappings on $\left(\Phi_{H \oplus F}\right)^{\times}$.

As the representation of $s u(2,2)$ is irreducible the UEA is the space of continuous operators acting on the RHS $\Phi_{H \oplus F} \subset \mathcal{H}_{H \oplus F} \subset \Phi_{H \oplus F}^{\times}$. Note that the UEA has a basis made of the monomials

$$
\mathcal{T}=\mathcal{A}_{+}^{\alpha} \mathcal{B}_{+}^{\beta} \mathcal{C}_{+}^{\gamma} \mathcal{D}_{+}^{\delta} \mathcal{E}_{+}^{\varepsilon} \mathcal{F}_{+}^{\zeta} \mathcal{J}^{\eta} \mathcal{M}^{\theta} \mathcal{Q}^{\iota} \mathcal{F}_{-}^{\zeta^{\prime}} \mathcal{E}_{-}^{\varepsilon^{\prime}} \mathcal{D}_{-}^{\delta^{\prime}} \mathcal{C}_{-}^{\gamma^{\prime}} \mathcal{B}_{-}^{\beta^{\prime}} \mathcal{A}_{-}^{\alpha^{\prime}}
$$

and it can be splitted in two subsets $U E A=U E A_{1} \oplus U E A_{2}$, where $U E A_{1}$ and $U E A_{2}$ are spanned by the operators $\mathcal{T}_{1}$ and $\mathcal{T}_{2}$, respectively, such that

$$
\gamma+\delta+\varepsilon+\zeta+\zeta^{\prime}+\varepsilon^{\prime}+\delta^{\prime}+\gamma^{\prime}=\left\{\begin{array}{cc}
\text { even } & \text { for } \mathcal{T}_{1} \\
\text { odd } & \text { for } \mathcal{T}_{2}
\end{array}\right.
$$

Thus, $U E A_{1}$ (that is an algebra) is the space of continuous operators acting on the RHS $\Phi_{H} \subset \mathcal{L}_{H} \subset \Phi_{H}^{\times}$ and $\Phi_{F} \subset \mathcal{L}_{F} \subset \Phi_{F}^{\times}$, i.e, in the space of the Jacobi Harmonics and the Jacobi Fermion Harmonics, respectively. Indeed

$$
\mathcal{T}_{1} \Phi_{H} \subset \Phi_{H}, \mathcal{T}_{1}^{\dagger} \Phi_{H}^{\times} \subset \Phi_{H}^{\times} ; \quad \mathcal{T}_{1} \Phi_{F} \subset \Phi_{F}, \mathcal{T}_{1}^{\dagger} \Phi_{F}^{\times} \subset \Phi_{F}^{\times}
$$

However, $U E A_{2}$ mix the two RHS.

\section{B. Discrete and discrete-continuous bases in $\mathcal{L}_{H}$}

In Section IV] we have defined the continuous basis $|x,(m, q)\rangle$ for $m$ and $q$ fixed. Now, it is the time for extending these notions to changing values of $m$ and $q$. In this Subsection we will discuss only the case of integer values of $(j, m, q)$ related to the Jacobi Harmonics. The analysis of the case with half-integer parameters is completely similar and it will be omitted.

Let $\Xi_{H}$ be the space of functions $f(x, \phi, \chi) \in \mathcal{L}_{H}$,

$$
f(x, \phi, \chi)=\sum_{j=0}^{\infty} \sum_{m=-j}^{j} \sum_{q=-j}^{j} f_{m, q}^{j} \mathcal{N}_{j}^{m, q}(x, \phi, \chi),
$$

such that for any $r, s=0,1,2, \ldots$, the following series converge:

$$
t_{r, s}(f):=\sum_{j, m, q}\left|f_{m, q}^{j}\right|(j+|m|+1)^{r}(j+|q|+1)^{s} .
$$


Due to (42) and (56), the convergence of the series (55) is absolute and uniform due to the Wiesstrass M-Theorem, and hence pointwise. Using arguments similar to those in Section IV we conclude that $\Xi_{H} \subset \mathcal{L}_{H} \subset \Xi_{H}^{\times}$is a RHS. We also have that $\Xi_{H} \subset \Phi_{H} \subset \mathcal{L}_{H} \subset \Phi_{H}^{\times} \subset \Xi_{H}^{\times}$, where the canonical inclusions $\mathfrak{i}(f)=f$ are always continuous.

Next, let us consider an abstract infinite dimensional separable Hilbert space $\mathcal{H}_{H}$ and a unitary mapping $U: \mathcal{H}_{H} \longmapsto \mathcal{L}_{H}$. Take, $\Omega_{H} \equiv U^{-1} \Xi_{H}$ and $\Psi_{H} \equiv U^{-1} \Phi_{H}$ with the topology transported by $U^{-1}$. Then, we have an abstract RHS $\emptyset_{H} \subset \mathcal{H}_{H} \subset \varnothing_{H}^{\times}$related to $\Xi_{H} \subset \mathcal{L}_{H} \subset \Xi_{H}^{\times}$through the following the diagram

$$
\begin{aligned}
& \Omega_{H} \subset \Psi_{H} \subset \mathcal{H}_{H} \subset \Psi_{H}^{\times} \subset \Omega_{H}^{\times} \\
& \begin{array}{lllllll} 
& U & \downarrow & U \downarrow & U \downarrow & U \downarrow
\end{array} \quad \\
& \Xi_{H} \subset \Phi_{H} \subset \mathcal{L}_{H} \subset \Phi_{H}^{\times} \subset \Xi_{H}^{\times}
\end{aligned}
$$

Now, we generalize the kets $|j,(m, q)\rangle$ (31) as follows

$$
|j, m, q\rangle:=U^{-1} \mathcal{N}_{j}^{m, q}(x, \phi, \chi) .
$$

From (48) the orthogonality and completeness relations (33) are now

$$
\left\langle j, m, q \mid j^{\prime}, m^{\prime}, q^{\prime}\right\rangle=\delta_{j, j^{\prime}} \delta_{m, m^{\prime}} \delta_{q, q^{\prime}}, \quad \sum_{j, m, q}|j, m, q\rangle\langle j, m, q|=\mathcal{I},
$$

where $\mathcal{I}$ is the identity on the space of the Jacobi Harmonics, $\mathcal{L}_{H}$.

Also for the elements $|x,(m, q)\rangle$ (with $x \in[-1,1]$ ) given by (34), we can remove the parenthesis and define

$$
|x, m, q\rangle:=\sum_{j}|j, m, q\rangle \mathbb{J}_{j}^{m, q}(x) .
$$

From (59) and (60) we obtain the relation

$$
\left\langle j, m^{\prime}, q^{\prime} \mid x, m, q\right\rangle=\left\langle x, m, q \mid j, m^{\prime}, q^{\prime}\right\rangle=\mathbb{J}_{j}^{m, q}(x) \delta_{m, m^{\prime}} \delta_{q, q^{\prime}},
$$

that shows that the $J_{j}^{m, q}(x)$ are the (real) transition matrices between both bases.

The action of $|x, m, q\rangle$ on an arbitrary $|f\rangle \in \Omega_{H}$ is also given by (44) but now considering besides the sum in $m$ and $q$. After (46), we show that $|x, m, q\rangle$ is anti-linear. Furthermore, it is also continuous on $\Omega_{H}$, since,

$$
\begin{aligned}
|\langle f \mid x, m, q\rangle| & \leq \sum_{j, m, q}\left|f_{m, q}^{j}\right|(j+|m|+1)^{2}(j+|q|+1) \\
& \leq \sum_{j, m, q}\left|f_{m, q}^{j}\right|(j+|m|+1)^{2}(j+|q|+1)=t_{2,1}(f) .
\end{aligned}
$$

Again from (10), (59) and (60) we easily see that

$$
\left\langle x^{\prime}, m^{\prime}, q^{\prime} \mid x, m, q\right\rangle=\delta\left(x-x^{\prime}\right) \delta_{m, m^{\prime}} \delta_{q, q^{\prime}},
$$

On the other hand note that we can rewrite (61) using (62) as

$$
\begin{aligned}
\left\langle x, m, q \mid j, m^{\prime}, q^{\prime}\right\rangle & =\int_{-1}^{1} \delta\left(x-x^{\prime}\right) \delta_{m, m^{\prime}} \delta_{q, q^{\prime}} \mathbb{J}_{j}^{m, q}\left(x^{\prime}\right) d x^{\prime} \\
& =\int_{-1}^{1}\left\langle x, m, q \mid x^{\prime}, m^{\prime}, q^{\prime}\right\rangle \mathbb{J}_{j}^{m, q}\left(x^{\prime}\right) d x^{\prime}
\end{aligned}
$$

and if we omit the arbitrary ket, $\langle x, m, q|$, we recover the generalization of (36), i.e.

$$
|j, m, q\rangle=\int_{-1}^{1}|x, m, q\rangle \mathbb{J}_{j}^{m, q}(x) d x,
$$


Next, take an arbitrary $f \in \Psi_{H}$ and with the help of (63) we get

$$
\begin{aligned}
\left\langle j^{\prime}, m^{\prime}, q^{\prime} \mid f\right\rangle & =\sum_{j, m, q} f_{m, q}^{j}\left\langle j^{\prime}, m^{\prime}, q^{\prime} \mid j, m, q\right\rangle \\
& =\sum_{j, m, q} f_{m, q}^{j} \int_{-1}^{1}\left\langle j^{\prime}, m^{\prime}, q^{\prime} \mid x, m, q\right\rangle \mathbb{J}_{j}^{m, q}(x) d x \\
& =\sum_{m, q} \int_{-1}^{1}\left\langle j^{\prime}, m^{\prime}, q^{\prime} \mid x, m, q\right\rangle \sum_{j} f_{m, q}^{j} \mathbb{J}_{j}^{m, q}(x) d x \\
& =\sum_{m, q} \int_{-1}^{1}\left\langle j^{\prime}, m^{\prime}, q^{\prime} \mid x, m, q\right\rangle f^{m, q}(x) d x
\end{aligned}
$$

where the expression for $f^{m, q}(x)$ is obvious after (44). We have used the Lebesgue theorem in order to insert the sum inside the integral from (42) and (56), Same expressions guarantee the absolute convergence of the resulting series, so that we may change the order of summation. If we omit the arbitrary bra $\left\langle j^{\prime}, m^{\prime}, q^{\prime}\right|$, we obtain the following expression:

$$
|f\rangle=\sum_{m, q} \int_{-1}^{1} d x|x, m, q\rangle f^{m, q}(x)
$$

which generalizes (38). From (65) and (44), we have that for $|f\rangle \in \Omega_{H}$,

$$
|f\rangle=\sum_{m, q} \int_{-1}^{1} d x|x, m, q\rangle f^{m, q}(x)=\sum_{m, q} \int_{-1}^{1} d x|x, m, q\rangle\langle x, m, q \mid f\rangle,
$$

and we obtain the completeness identity

$$
\sum_{m, q} \int_{-1}^{1} d x|x, m, q\rangle\langle x, m, q|=\mathcal{I}
$$

where $\mathcal{I}$ is the canonical injection $\mathcal{I}: \Omega_{H} \longmapsto \Omega_{H}^{\times}$. Note that some of the proofs of the previous results are albeit formal.

Finally, the operators $\mathcal{J}, \mathcal{M}, \mathcal{Q}, \mathcal{A}_{ \pm}, \mathcal{B}_{ \pm}$and $\mathcal{T}_{1}$ defined in (54) are continuous on $\Psi_{H}$ with formal adjoints having the same properties. In consequence, they can be extended into $\Psi_{H}^{\times}$as weakly continuous operators. In addition, with the aid of the relation (41), we show that these operators are continuous linear mappings from $\Omega_{H}$ into $\Phi_{H}$. The proof is simple. For instance for $\mathcal{A}_{+}$, we readily see that $p_{r, s}\left(\mathcal{A}_{+} f\right) \leq 2^{r / 2} t_{r+1, s}(f)$, for all $f \in \Omega_{H}$, proving this continuity.

Let us recall again that the discussion for half-integer parameters is analogous.

\section{The full continuous basis $\{|x, \phi, \chi\rangle\}$}

The next goal is to define and give some properties of the generalized eigenvectors of the form $|x, \phi, \chi\rangle$. By definition $\left[\mathcal{N}_{j}^{m, q}(x, \phi, \chi)\right.$ is the transition matrix between the bases $\{|j, m, q\rangle\}$ and $\{|x, \phi, \chi\rangle\}$, i.e., for arbitrary $|j, m, q\rangle \in \Xi_{H}$ we have

$$
\langle x, \phi, \chi \mid j, m, q\rangle:=\mathcal{N}_{j}^{m, q}(x, \phi, \chi) .
$$


If $|f\rangle=\sum_{j=0}^{\infty} \sum_{m, q} f_{m, q}^{j}|j, m, q\rangle \in \Xi_{H}$, one has

$$
\begin{aligned}
|\langle f \mid x, \phi, \chi\rangle| & \leq \sum_{j, m, q}\left|f_{m, q}^{j}\right|\left|\mathcal{N}_{j}^{m, q}(x, \phi, \chi)\right| \leq \sum_{j, m, q}\left|f_{m, q}^{j}\right| \sqrt{j+1 / 2}\left|\mathcal{J}_{j}^{m, q}(x)\right| \\
& \leq \sum_{j, m, q}\left|f_{m, q}^{j}\right| \sqrt{j+1 / 2}(j+|m|+1)(j+|q|+1) \\
& \leq \sum_{j, m, q}\left|f_{m, q}^{j}\right|(j+|m|+1)^{2}(j+|q|+1)=t_{2,1}(|f\rangle) .
\end{aligned}
$$

The anti-linearity of $|x, \phi, \chi\rangle$ on $\Omega_{H}$ is obvious and the latter chain of inequalities shows that $|x, \phi, \chi\rangle$ is also continuous on $\Omega_{H}$, so that $|x, \phi, \chi\rangle \in \Omega_{H}^{\times}$.

In Appendix B (90) we have proven that $e^{-i \phi}(\cos \chi+i X \sin \chi)$ is a continuous mapping in $\Phi_{H, F}$ for each value of $\phi$ and $\chi$.

On each function $f(x, \phi, \chi)$ in the Hilbert spaces $L_{H}$, one may define the following bounded operators:

$$
\begin{aligned}
X f_{H, F}(x, \phi, \chi) & =x f_{H, F}(x, \phi, \chi), & \Phi f_{H, F}(x, \phi, \chi) & =\phi f_{H, F}(x, \phi, \chi), \\
\cos \chi f_{H, F}(x, \phi, \chi) & =\cos \chi f_{H, F}(x, \phi, \chi), & \sin \chi f_{H, F}(x, \phi, \chi) & =\sin \chi f_{H, F}(x, \phi, \chi) .
\end{aligned}
$$

Then, following the same lines as in Appendix B, one finds that the operator

$$
e^{-i \Phi}(\cos \chi+i X \sin \chi)
$$

is continuous on $\Xi_{H}$. Therefore, the formal adjoint is continuous on the weak anti-dual, $\Xi_{H}^{\times}$. Consequently, by means of the operator $U$, defined in (57) we get that

$$
R=U^{-1} e^{-i \Phi}(\cos \chi+i X \sin \chi) U,
$$

is a continuous operator on $\Omega_{H}$ and its formal adjoint is weakly continuous on $\Omega_{H}^{\times}$, so that

$$
R^{\dagger}|x, \phi, \chi\rangle=e^{i \phi}(\cos \chi-i x \sin \chi)|x, \phi, \chi\rangle,
$$

for each $x \in[-1,1]$ and each value of the angles $\phi$ and $\chi$.

From (66), (48) and the completeness relation of (59) we easily obtain

$$
\left\langle x^{\prime}, \phi^{\prime}, \chi^{\prime} \mid x, \phi, \chi\right\rangle=\delta\left(x-x^{\prime}\right) \delta\left(\phi-\phi^{\prime}\right) \delta\left(\chi-\chi^{\prime}\right),
$$

and we immediately have that

$$
|j, m, q\rangle=\int \mathcal{N}_{j}^{m, q}(x, \phi, \chi)|x, \phi, \chi\rangle d x d \phi d \chi .
$$

If we sustitute $\mathcal{N}(x, \phi, \chi)$ for its expression of (66) we get

$$
|j, m, q\rangle=\int|x, \phi, \chi\rangle\langle x, \phi, \chi \mid j, m, q\rangle d x d \phi d \chi
$$

so that

$$
\int|x, \phi, \chi\rangle\langle x, \phi, \chi| d x d \phi d \chi=\mathcal{I},
$$

which is the canonical injection $\mathcal{I}: \Omega_{H} \longmapsto \Omega_{H}^{\times}$. Next, recalling the completeness relation (59) where $I$ is the identity on either $\mathcal{L}_{H}$, we have formally for each value of $x, \phi$ and $\chi$ that

$$
|x, \phi, \chi\rangle=\sum_{j, m, q}|j, m, q\rangle\langle j, m, q \mid x, \phi, \chi\rangle=\sum_{j, m, q}\left[\mathcal{N}_{j}^{m, q}(x, \phi, \chi)\right]^{*}|j, m, q\rangle .
$$

\section{CONCLUDING REMARKS}

The set of Algebraic Jacobi Functions, $\left\{\mathcal{J}_{j}^{m, q}(x)\right\}$ as well as the set of Normalized Algebraic Jacobi
Functions $\left\{\mathbb{J}_{j}^{m, q}(x)\right\}$, support a non-unitary and 
non-irreducible representation for the Lie algebra $s u(2,2)$. However, $\left\{\mathbb{J}_{j}^{m, q}(x)\right\}_{j \geq \sup (|m|,|q|)}^{m, q(\text { fixed })}$ constitutes an orthonormal basis for the Hilbert space $L^{2}[-1,1]$ and supports a UIR of $s u(1,1)$. These bases span dense subspaces of $L^{2}[-1,1]$ having their own locally convex topology, stronger than the Hilbert space topology. Each of the subspaces is characterized by fixed values of $m$ and $q$, so that we may denote them as $\Phi_{m, q}$. The triplets $\Phi_{m, q} \subset L^{2}[-1,1] \subset \Phi_{m, q}^{\times}$form RHS or Gelfand triplets, $\Phi_{m, q}^{\times}$being the dual of $\Phi_{m, q}$. Each of the spaces $\Phi_{m, q}$ support a representation of the Lie algebra $s u(1,1)$ by continuous operators on $\Phi_{m, q}$, where the continuity is respect to the locally convex topology of these spaces. These operators may be extended by duality to continuous operators on the duals $\Phi_{m, q}^{\times}$, so that we have different and nonequivalent representations of the algebra $s u(1,1)$ by continuous operators.

Algebraic Jacobi Functions are also related to different pairs of creation and annihilation ladder operators transforming AJF into others with different values of the parameters $m$ and $q$. Consequently, these ladder operators should be transformations between different spaces of the family $\Phi_{m, q}$. This is really what happens, the ladder operators being continuous as mappings between these spaces. These continuity induce similar relations between the duals.

Up to this point, we have used a concrete realization of RHS using Algebraic Jacobi Functions. For each of these concrete realizations, we may construct an abstract RHS, $\Psi_{m, q} \subset \mathcal{H} \subset \Psi_{m, q}^{\times}$unitarily equivalent to the original $\Phi_{m, q} \subset L^{2}[-1,1] \subset$ $\Phi_{m, q}^{\times}$. This abstract representation permits to span each of the function on the space $\Phi_{m, q}$, for whatever values of $m$ and $q$, in terms of continuous basis of vectors on the dual, $\Psi_{m, q}^{\times}$, of the abstract space, $\Psi_{m, q}$, unitarily equivalent to $\Phi_{m, q}$. These continuous bases are eigenvectors of some continuous operators defined on $\Psi_{m, q}$.

Along this continuous basis, we always have a discrete orthonormal basis in terms of which all vectors on the spaces $\Phi_{m, q}$ and $\Psi_{m, q}$ may be written.
Since these spaces are contained in their respective duals, these orthonormal basis are also contained in the duals, so that both discrete and continuous basis coexist in the same dual spaces. Then, we have established the relation between discrete and continuous basis in our example and have shown that brackets among vectors of these two different basis are well defined and can be given in terms of the AJF.

Remark that we have used two non-equivalent choices for the locally convex topology of the spaces spanned by the AJF with fixed values of $m$ and $q$ and therefore, we have two non-equivalent triplets. These spaces, $\Phi_{m, q}$ and $\Xi_{m, q}$, have not only different topologies, they are even different as linear spaces of functions, although one contains the other as subspace $\left(\Xi_{m, q} \subset \Phi_{m, q}\right)$. Furthermore, some particular properties are different in each case. These spaces are defined by series of functions that always converge pointwise in one case $\left(\Xi_{m, q}\right)$ and not in the other. Nevertheless, the properties of the operators representing the algebras $s u(2,2)$ and $s u(1,1)$, such as continuity as well as the properties of the ladder operators are similar in both triplets.

So far, we have obtained RHS for fixed values of $m$ and $q$. However by introducing Jacobi Harmonic we have been able to construct a RHS that include all values of the parameters. This construction allows also to split this RHS into two different triplets, one for integer and the other for half-integer values of $j$. On these spaces, we may define all the objects treated along the present article and continuity properties may be also proved. In this context, we have discussed the behaviour and properties of ladder operators and continuous and discrete bases, relations between both triplets, etc., pointing out the relevant role played by the Lie algebra and its UEA.

A possible physical applications of AJF could be to describe quantum systems of two particles with the same spin because for $j$ fixed they are described by the representation $D_{j} \otimes D_{j}$ of $S U(2) \otimes S U(2)$ (see (11)).

\section{ACKNOWLEDGEMENTS}

We acknowledge partial financial support to the Spanish MINECO, grant MTM2014-57129-C2-1-P, and the Junta de Castilla y León, grants VA137G18 and BU229P18. 


\section{APPENDIX A: PROPERTIES OF THE ALGEBRAIC JACOBI FUNCTIONS.}

The AJF (2) are either pure polynomials or "quasi-polynomials" in $x$. Indeed

$$
\begin{array}{ll}
j, m, q \in \mathbb{Z}: & \mathcal{J}_{j}^{m, q}(x)=\left\{\begin{aligned}
P(x) & \text { if } m-q \text { even } \\
\sqrt{1-x^{2}} P(x) & \text { if } m-q \text { odd }
\end{aligned}\right. \\
j, m, q \in \mathbb{Z}+1 / 2: & \mathcal{J}_{j}^{m, q}(x)= \begin{cases}\sqrt{1-x} P(x) & \text { if } m-q \text { even } \\
\sqrt{1+x} P(x) & \text { if } m-q \text { odd }\end{cases}
\end{array}
$$

where $P(x)$ are appropriate polynomials in $x$.

We mention in Section $\llbracket A$ that the AJF $\mathcal{J}_{j}^{m, q}(x)$ have symmetry properties in $(m, q, x)$ related to the Jacobi differential equation (6) which is invariant under $(m, q, x) \leftrightarrow(q, m, x),(m, q, x) \leftrightarrow(-m,-q, x)$, $(m, q, x) \leftrightarrow(m,-q,-x)$ and their combinations.

We can obtain the following explicit expression for the $\mathcal{J}_{j}^{m, q}(x)$ (3)

$$
\mathcal{J}_{j}^{m, q}(x)=K[j, m, q] \sum_{s=0}^{j-m} \frac{(-1)^{j-m-s}\left(\frac{1-x}{2}\right)^{j+\frac{q-m}{2}-s}\left(\frac{1+x}{2}\right)^{\frac{m-q}{2}+s}}{\Gamma(s+1) \Gamma(j-m-s+1) \Gamma(j+q-s+1) \Gamma(m-q+s+1)},
$$

such that

$$
K[j, m, q]:=\sqrt{\Gamma(j+m+1) \Gamma(j-m+1) \Gamma(j+q+1) \Gamma(j-q+1)} .
$$

The expression of $K[j, m, q]$ is invariant under the above mentioned changes interchanges of $m$ and $q$. From (77) $\mathcal{J}_{j}^{q, m}(x)$ reads as follows

$$
\mathcal{J}_{j}^{q, m}(x)=K[j, m, q] \sum_{s=0}^{j-q} \frac{(-1)^{j-q-s}\left(\frac{1-x}{2}\right)^{j+\frac{m-q}{2}-s}\left(\frac{1+x}{2}\right)^{\frac{q-m}{2}+s}}{\Gamma(s+1) \Gamma(j-q-s+1) \Gamma(j+m-s+1) \Gamma(q-m+s+1)} .
$$

Comparing with expression (77) of $\mathcal{J}_{j}^{m, q}(x)$ we see that with the change $s=q-m+t$ we transform (77) in

$$
\mathcal{J}_{j}^{m, q}(x)=K[j, m, q] \sum_{t=m-q}^{j-q} \frac{(-1)^{j-q-t}\left(\frac{1-x}{2}\right)^{j+\frac{m-q}{2}-t}\left(\frac{1+x}{2}\right)^{\frac{q-m}{2}+t}}{\Gamma(q-m+t+1) \Gamma(j-q-t+1) \Gamma(j+m-t+1) \Gamma(t+1)},
$$

Now let us consider the possible values of $m-q$. If $m-q \geq 0$ (i.e., $m \geq q$ ) we observe that $q-m+t+1 \leq 0$ for $t=0,1, \cdots, m-q-1$ then $|\Gamma(q-m+t+1)|=\infty$ and these terms in (79) shall become zero. In the opposite case of $m-q \leq 0$ (i.e., $m \leq q$ ) we see $t+1 \leq 0$ for $t=m-q, m-q+1, \cdots,-1$ and hence $|\Gamma(t+1)|=\infty$. So, in both cases we can consider the sum for $t=0,1, \cdots, j-q$ recovering the expression (78) for $\mathcal{J}_{j}^{q, m}(x)$. Then we have proved that $\mathcal{J}_{j}^{m, q}(x)=\mathcal{J}_{j}^{q, m}(x)$.

Now we study the case of $\mathcal{J}_{j}^{-m,-q}(x)$ which expression from (77) is

$$
\mathcal{J}_{j}^{-m,-q}(x)=K[j, m, q] \sum_{s=0}^{j+m} \frac{(-1)^{j+m-s}\left(\frac{1-x}{2}\right)^{j+\frac{-q+m}{2}-s}\left(\frac{1+x}{2}\right)^{\frac{-m+q}{2}+s}}{\Gamma(s+1) \Gamma(j+m-s+1) \Gamma(j-q-s+1) \Gamma(-m+q+s+1)}
$$

Comparing this expression with that of $\mathcal{J}_{j}^{q, m}(x)$ (178) we see that both coincide up a global sign $(-1)^{q+m}$ and the upper limit of the sum that is $j+m$ instead $j-q$. But considering, for instance, that $j+m \geq j-q$ we see that $|\Gamma(j-q-s+1)|=\infty$ for $s=j-q+1, j-q+2, \cdots, j+m$. In the opposite case $j+m \leq j-q$ we have $|\Gamma(j+m-s+1)|=\infty$ for $s=j+m+1, j+m+2, \cdots, j-q$. So, in both cases we can consider the upper limit of the sum $s-j-q$ and we have proved $\mathcal{J}_{j}^{-m,-q}(x)=(-1)^{m+q} \mathcal{J}_{j}^{q, m}(x)$.. 
On the other hand, from the inspection of the Jacobi differential equation (6) we see that under the change $m \rightarrow-m$ (or $q \rightarrow-q, m \leftrightarrow-q$ ) we recover the equation (6) if we also perform the change of sign of the variable $x$, i.e., $x \rightarrow-x$. For instance, $\mathcal{J}_{j}^{m,-q}(-x)$ is solution of (6) and it will be written as

$$
\mathcal{J}_{j}^{m,-q}(-x):=K[j, m, q] \sum_{s=0}^{j-m} \frac{(-1)^{j-m-s}\left(\frac{1+x}{2}\right)^{j+\frac{-q-m}{2}-s}\left(\frac{1-x}{2}\right)^{\frac{m+q}{2}+s}}{\Gamma(s+1) \Gamma(j-m-s+1) \Gamma(j-q-s+1) \Gamma(m+q+s+1)} .
$$

Using the change $s=j-m-t$ we rewrite the previous expressions as

$$
\mathcal{J}_{j}^{m,-q}(-x)=K[j, m, q] \sum_{t=0}^{j-m} \frac{(-1)^{t}\left(\frac{1+x}{2}\right)^{\frac{m-q}{2}+t}\left(\frac{1-x}{2}\right)^{j+\frac{q-m}{2}+t}}{\Gamma(j-m-t+1) \Gamma(t+1) \Gamma(m-q+t+1) \Gamma(j+q-t+1)} .
$$

Since $(-1)^{j-m-t}=(-1)^{j-m}(-1)^{-t}=(-1)^{j-m}(-1)^{t}$ we see that $\mathcal{J}_{j}^{m,-q}(-x)=(-1)^{j-m} \mathcal{J}_{j}^{m, q}(x)$. Resuming we have for all $m$ and $q$

$$
\begin{aligned}
& \mathcal{J}_{j}^{q, m}(x)=\mathcal{J}_{j}^{m, q}(x) \\
& J_{j}^{-m,-q}(x)=(-1)^{m+q} \mathcal{J}_{j}^{m, q}(x), \\
& \mathcal{J}_{j}^{m,-q}(-x)=(-1)^{j-m} \mathcal{J}_{j}^{m, q}(x),
\end{aligned}
$$

and their combinations.

\section{APPENDIX B: MULTIPLICATION OPERATORS.}

In [23], we have analyzed the behaviour of the multiplication operator on spaces including Spherical Harmonics. Because the relations between Spherical Harmonics and Jacobi Harmonics we study now the action of the multiplication operator on spaces of Jacobi functions.

Starting from the definition of the functions $\mathcal{J}_{j}^{m, q}(x)$ given in (4), and the property for the Jacobi polynomials given in [53] (p. 782, eq. 22.7.15) as

$$
(1-x)\left(n+\frac{\alpha}{2}+\frac{\beta}{2}+1\right) J_{n}^{\alpha+1, \beta}(x)=(n+\alpha+1) J_{n}^{\alpha, \beta}(x)-(n+1) J_{n+1}^{\alpha, \beta}(x),
$$

we obtain the following properties of the multiplication operator $X f(x)=x f(x)$ on the $\operatorname{AJF} \mathcal{J}_{j}^{m, q}(x)$ :

$$
x \mathcal{J}_{j}^{m, q}(x)=\mathcal{J}_{j}^{m, q}(x)+c_{j}^{m, q} \mathcal{J}_{j-1}^{m-1, q-1}(x)+d_{j}^{m, q} \mathcal{J}_{j}^{m-1, q-1}(x)+e_{j}^{m, q} \mathcal{J}_{j+1}^{m-1, q-1}(x),
$$

with

$$
\begin{aligned}
c_{j}^{m, q} & =-\frac{\sqrt{(j+m-1)(j+m)(j+q-1)(j+q)}}{j(2 j+1)}, \\
d_{j}^{m, q} & =\frac{\sqrt{(j-m+1)(j+m)(j-q+1)(j+q)}}{j(j+1)(2 j+1)}, \\
e_{j}^{m, q} & =-\frac{\sqrt{(j-m+1)(j-m+2)(j-q+1)(j-q+2)}}{(j+1)(2 j+1)} .
\end{aligned}
$$

In the case of the functions $\mathbb{J}_{j}^{m, q}(x)$ defined in (9) expression (82) becomes

$$
\begin{aligned}
x \mathbb{J}_{j}^{m, q}(x)=\mathbb{J}_{j}^{m, q}(x) & +c_{j}^{m, q} \sqrt{\frac{j+1 / 2}{j-1 / 2}} \mathbb{J}_{j-1}^{m-1, q-1}(x) \\
& +d_{j}^{m, q} \mathbb{J}_{j}^{m-1, q-1}(x)+e_{j}^{m, q} \sqrt{\frac{j+1 / 2}{j+1 / 2}} \mathbb{J}_{j+1}^{m-1, q-1}(x) .
\end{aligned}
$$


Now we have to look for the spaces where the multiplication operator $X$ is well defined and continuous. Obviously, $X$ mixes up the spaces $\Xi_{m, q}$ and also spaces $\Phi_{m, q}$, so that we need another kind of space. The fact that $X$ conserves the parity of $j, m$ and $q$ gives us an interesting clue for the definition of these spaces.

Let us consider two Hilbert spaces, one for $j$ integer and the other for $j$ half-integer that we denote here as $\mathcal{G}_{H}$ and $\mathcal{G}_{F}$, respectively. Thus, $\mathcal{G}_{H}$ is the space of all series of the form:

$$
f(x):=\sum_{j=0}^{\infty} \sum_{m, q=-j}^{j} f_{j}^{m, q} \mathbb{J}_{j}^{m, q}(x), \quad \text { with } \quad \sum_{j=0}^{\infty} \sum_{m, q=-j}^{j}\left|f_{j}^{m, q}\right|^{2}<\infty,
$$

and a similar definition for $j$ half-integer (in this case the sum goes from $j=1 / 2$ to $\infty$ ). These spaces may be written as infinite direct sums:

$$
\mathcal{G}_{H}=\bigoplus_{|m|,|q|=0}^{\infty} \mathcal{G}_{|m|,|q|}, \quad \mathcal{G}_{F}=\bigoplus_{|m|,|q|=1 / 2}^{\infty} \mathcal{G}_{|m|,|q|},
$$

where $\mathcal{G}_{|m|,|q|}$ are copies of $L^{2}[-1,1]$. Then, let us define the space $\Delta_{H}$ as the space of all series in $\mathcal{G}_{H}$ such that

$$
\|f\|_{r, s}^{2}:=\sum_{j=0}^{\infty} \sum_{m, q=-j}^{j}\left|f_{j}^{m, q}\right|^{2}(j+|m|+1)^{2 r}(j+|q|+1)^{2 s}<\infty,
$$

for all $r, s=0,1,2, \ldots$ Analogously, we define $\Delta_{F}$ for $j$ half-integer.

Then, the multiplication operator $X$ is continuous on both $\Delta_{H}$ and $\Delta_{F}$. This means that both $\Delta_{H, F}$ reduce $X$, i.e., $X \Delta_{H} \subset \Delta_{H}$ and $X \Delta_{F} \subset \Delta_{F}$ and that $X$ is a continuous linear mapping on $\Delta_{H, F}$. Let us proceed for the proof for $\Delta_{H}$ (the proof for $\Delta_{F}$ being identical). Observe that the two first coefficients in (83), $c_{j}^{m, q}$ and $d_{j}^{m, q}$, have a $j$ in the denominator, which may be a problem for $j=0$. This is not the case, since after (82) and (83), we have that

$$
c_{0}^{m, q}=d_{0}^{m, q}=0,
$$

or in other words

$$
\begin{aligned}
& X \mathcal{J}_{0}^{0,0}(x)=x \mathcal{J}_{0}^{0,0}(x)=\mathcal{J}_{0}^{0,0}(x)-\sqrt{2} \mathcal{J}_{1}^{-1,-1}(x), \\
& X \mathbb{J}_{0}^{0,0}(x)=x \mathbb{J}_{0}^{0,0}(x)=\mathbb{J}_{0}^{0,0}(x)-\frac{2 \sqrt{2}}{\sqrt{3}} \mathbb{J}_{1}^{-1,-1}(x) .
\end{aligned}
$$

So that, for $j=0$, the coefficients having a factor $j$ in the denominator do not appear. Then, for any series (85) in $\Delta_{H}$, we have that

$$
\begin{aligned}
(X f)(x)= & X\left[\sum_{j=0}^{\infty} \sum_{m, q=-j}^{j} f_{j}^{m, q} \mathbb{J}_{j}^{m, q}(x)\right]=\sum_{j=0}^{\infty} \sum_{m, q=-j}^{j} f_{j}^{m, q} x \mathbb{J}_{j}^{m, q}(x) \\
= & \sum_{j, m, q} f_{j}^{m, q} \mathbb{J}_{j}^{m, q}(x)+\sum_{j, m, q} \sqrt{\frac{j+1 / 2}{j-1 / 2}} f_{j}^{m, q} c_{j}^{m, q} \mathbb{J}_{j-1}^{m-1, q-1}(x) \\
& +\sum_{j, m, q} f_{j}^{m, q} d_{j}^{m, q} \mathbb{J}_{j}^{m-1, q-1}(x)+\sum_{j, m, q} \sqrt{\frac{j+1 / 2}{j+3 / 2}} f_{j}^{m, q} e_{j}^{m, q} \mathbb{J}_{j+1}^{m-1, q-1}(x) .
\end{aligned}
$$

Since $j+|m|+2 \leq 2(j+|m|+1)$, all coefficients (83) are bounded by $2(j+|m|+1)(j+|q|+1)$ (recall above comments for the case $j=0$ ) and taking into account (86) and (43) we have that

$$
\begin{aligned}
\|X f\|_{r, s}^{2} & \leq 16 \sum_{j=0}^{\infty} \sum_{m, q=-j}^{j}\left|f_{j}^{m, q}\right|^{2}(j+1 / 2)(j+|m|+1)^{2(r+1)}(j+|q|+1)^{2(s+1)} \\
& \leq 16 \sum_{j=0}^{\infty} \sum_{m, q=-j}^{j}\left|f_{j}^{m, q}\right|^{2}(j+|m|+1)^{2(r+2)}(j+|q|+1)^{2(s+1)}=16\|f\|_{r+2, s+1}^{2} .
\end{aligned}
$$


This proves both, that $X$ preserves $\Delta_{H}, X \Delta_{H} \subset \Delta_{H}$ and that $X$ is continuous on $\Delta_{H}$. Same proof is valid for $\Delta_{F}$.

Next, let us see what we may say in relation with the spaces spanned by the functions $\mathcal{N}_{j}^{m, q}(x, \phi, \chi)$ and the multiplication operator $X$. First of all, we have to remark that by the symmetry property under the interchange $\alpha \leftrightarrow \beta$ that reads

$$
J_{n}^{\beta, \alpha}(x)=(-1)^{n} J_{n}^{\alpha, \beta}(-x)
$$

Then eq. (81) may be also written as

$$
(1+x)\left(n+\frac{\alpha}{2}+\frac{\beta}{2}+1\right) J_{n}^{\alpha, \beta+1}(x)=(n+\beta+1) J_{n}^{\alpha, \beta}(x)+(n+1) J_{n+1}^{\alpha, \beta}(x) .
$$

Note that (87) appears also in Ref. 53] (p. 782, eq. 22.7.16). From (87), we readily obtain the following recurrence relation for the AJF:

$$
x \mathcal{J}_{j}^{m, q}(x)=-\mathcal{J}_{j}^{m, q}(x)+\hat{c}_{j}^{m, q} \mathcal{J}_{j-1}^{m-1, q+1}(x)+\hat{d}_{j}^{m, q} \mathcal{J}_{j}^{m-1, q+1}(x)+\hat{e}_{j}^{m, q} \mathcal{J}_{j+1}^{m-1, q+1}(x),
$$

with

$$
\begin{aligned}
& \hat{c}_{j}^{m, q}=\frac{\sqrt{(j+m-1)(j+m)(j-q-1)(j-q)}}{j(2 j+1)}, \\
& \hat{d}_{j}^{m, q}=\frac{\sqrt{(j-m+1)(j+m)(j-q)(j+q+1)}}{j(j+1)(2 j+1)}, \\
& \hat{e}_{j}^{m, q}=\frac{\sqrt{(j-m+1)(j-m+2)(j+q+1)(j+q+2)}}{(j+1)(2 j+1)} .
\end{aligned}
$$

The corresponding version of the expression (88) for the $\mathrm{NAJF} \mathbb{J}_{j}^{m, q}(x)$ is

$$
\begin{aligned}
x \mathbb{J}_{j}^{m, q}(x)=-\mathbb{J}_{j}^{m, q}(x) & +\hat{c}_{j}^{m, q} \sqrt{\frac{j+1 / 2}{j-1 / 2}} \mathbb{J}_{j-1}^{m-1, q+1}(x) \\
& +\hat{d}_{j}^{m, q} \mathbb{J}_{j}^{m-1, q+1}(x)+\hat{e}_{j}^{m, q} \sqrt{\frac{j+1 / 2}{j+1 / 2}} \mathbb{J}_{j+1}^{m-1, q+1}(x) .
\end{aligned}
$$

From (82) and (88), we obtain that

$$
\begin{aligned}
X \mathcal{J}_{j}^{m, q}(x)=x \mathcal{J}_{j}^{m, q}(x)= & \left.\frac{c_{j}^{m, q}}{2} \mathcal{J}_{j-1}^{m-1, q-1}(x)+\frac{\hat{c}_{j}^{m, q}}{2} \mathcal{J}_{j-1}^{m-1, q+1}(x)+\frac{d_{j}^{m, q}}{2} \mathcal{J}_{j}^{m-1, q-1}(x)\right) \\
& +\frac{\hat{d}_{j}^{m, q}}{2} \mathcal{J}_{j}^{m-1, q+1}(x)+\frac{e_{j}^{m, q}}{2} \mathcal{J}_{j+1}^{m-1, q-1}(x)+\frac{\hat{e}_{j}^{m, q}}{2} \mathcal{J}_{j+1}^{m-1, q+1}(x) .
\end{aligned}
$$

Then, let us use the definition of the functions $\mathcal{N}_{j}^{m, q}(x, \phi, \chi)$ as given in (47) and (84), so as to obtain the following relation:

$$
\begin{aligned}
& e^{-i \phi} e^{-i \chi}(1-x) \mathcal{N}_{j}^{m, q}(x, \phi, \chi) \\
& =\sqrt{\frac{j+1 / 2}{j-1 / 2}} c_{j}^{m, q} \mathcal{N}_{j-1}^{m-1, q-1}(x, \phi, \chi)+d_{j}^{m, q} \mathcal{N}_{j}^{m-1, q-1}(x, \phi, \chi)+\sqrt{\frac{j+1 / 2}{j+3 / 2}} e_{j}^{m, q} \mathcal{N}_{j+1}^{m-1, q-1}(x, \phi, \chi) .
\end{aligned}
$$

Now, it is rather easy to show that the operator $e^{-i \phi} e^{-i \chi}(1-X)$ is a continuous linear operator on $\Phi_{H}$ and $\Phi_{F}$ as defined in Subsection $\mathrm{VA}$. Similarly, we may find another similar relation such as

$$
\begin{aligned}
& e^{-i \phi} e^{i \chi}(1+x) \mathcal{N}_{j}^{m, q}(x, \phi, \chi) \\
& =\sqrt{\frac{j+1 / 2}{j-1 / 2}} \hat{c}_{j}^{m, q} \mathcal{N}_{j-1}^{m-1, q+1}(x, \phi, \chi)+\hat{d}_{j}^{m, q} \mathcal{N}_{j}^{m-1, q+1}(x, \phi, \chi)+\sqrt{\frac{j+1 / 2}{j+3 / 2}} \hat{e}_{j}^{m, q} \mathcal{N}_{j+1}^{m-1, q+1}(x, \phi, \chi),
\end{aligned}
$$


which gives the continuity of the operator $e^{-i \phi} e^{i \chi}(1+X)$ on both $\Phi_{H, F}$. Combining both last equations we obtain an interesting expression:

$$
\begin{aligned}
e^{-i \phi}(\cos \chi & +i x \sin \chi) \mathcal{N}_{j}^{m, q}(x, \phi, \chi) \\
= & \frac{1}{2}\left[\sqrt{\frac{j+1 / 2}{j-1 / 2}}\left(c_{j}^{m, q} \mathcal{N}_{j-1}^{m-1, q-1}(x, \phi, \chi)+\hat{c}_{j}^{m, q} \mathcal{N}_{j-1}^{m-1, q+1}(x, \phi, \chi)\right)\right. \\
& +d_{j}^{m, q} \mathcal{N}_{j}^{m-1, q-1}(x, \phi, \chi)+\hat{d}_{j}^{m, q} \mathcal{N}_{j}^{m-1, q+1}(x, \phi, \chi) \\
& \left.+\sqrt{\frac{j+1 / 2}{j+3 / 2}}\left(e_{j}^{m, q} \mathcal{N}_{j+1}^{m-1, q-1}(x, \phi, \chi)+\hat{e}_{j}^{m, q} \mathcal{N}_{j+1}^{m-1, q+1}(x, \phi, \chi)\right)\right],
\end{aligned}
$$

which shows that the operator $e^{-i \phi}(\cos \chi+i X \sin \chi)$ is continuous on $\Phi_{H, F}$ and, hence, its formal adjoint is extended to the duals $\Phi_{H, F}^{\times}$.

\section{APPENDIX C: INEQUALITIES FOR JACOBI FUNCTIONS.}

In formula (20) of Ref. [54] it is given an upper-bound for Jacobi polynomials that in our notation is

$$
\left|\mathcal{J}_{j}^{m, q}(x)\right| \leq\left[\frac{(j-m+1)(j+m+1)}{(j-q+1)(j+q+1)}\right]^{1 / 4},
$$

valid for $x \in[-1,1], m \geq 0, m \geq|q|$ and $j \geq|m|,|q|$. Moreover since

$$
(j-m+1)(j+m+1)-(j-q+1)(j+q+1)=-m^{2}+q^{2} \leq 0
$$

we see that

$$
\left|\mathcal{J}_{j}^{m, q}(x)\right| \leq 1, \quad \forall m \geq 0, m \geq|q|, j \geq|m|,|q| .
$$

In the following let us consider only those Jacobi functions $\mathcal{J}_{j}^{m, q}(x)$ such that $(j, m, q)$ verify that $m \geq q \geq$ 0 and $j \geq m, q$. Then using that $\mathcal{J}_{j}^{m, q}(x)=\mathcal{J}_{j}^{q, m}(x)$ as we have proved in (80) we see that inequality (92) is valid for all $m, q \geq 0$. Morever, according to $\mathcal{J}_{j}^{m, q}(x)=(-1)^{m+q} \mathcal{J}_{j}^{-m,-q}(x)$ (80) the inequality (92) is also valid for $(m, q)$ both negative numbers. Since the inequality (92) is valid for those $\mathcal{J}_{j}^{m,-q}(x)$ with $m \geq q$ applying the same reasoning using the equalities (80) we arrive to proved the the inequality (92) are valid for all $(j, m, q)$ verifying the conditions displayed in (5), i.e. $j \geq|m|,|q| ; 2 j \in \mathbb{N} ; j-m, j-q \in \mathbb{N}$. On the other hand the inequality (91) is valid for the same values of $(j, m, q)$ as (92) with some small changes and now it reads as follows

$$
\left|\mathcal{J}_{j}^{m, q}(x)\right| \leq\left[\frac{(j-a+1)(j+a+1)}{(j-b+1)(j+b+1)}\right]^{1 / 4}
$$

where $a=\max (|m|,|q|)$ and $b=\min (|m|,|q|)$.

As we mention before in Appendix A and taking into account the validity conditions of the (91) we see that we have enlarged the inequality (91) (or formula (20) of Ref. [54]) for all $\alpha$ and $\beta$ such that $n \geq|\alpha|,|\beta|$.

Finally for the sake of the requirements of this work we can write the following upper-bound for the $\mathcal{J}_{j}^{m, q}(x)$ from (93)

$$
\left|\mathcal{J}_{j}^{m, q}(x)\right| \leq(j+|m|+1)(j+|q|+1), \quad \forall j \geq|m|,|q| ; 2 j \in \mathbb{N} ; j-m, j-q \in \mathbb{N} .
$$

[1] E. Celeghini and M.A. del Olmo, Ann. Phys. 333, 90-103 (2013).
[2] E. Celeghini and M.A. del Olmo, Ann. Phys. 335, 
78-85 (2013).

[3] E. Celeghini, M.A. del Olmo and M.A. Velasco, J. Phys. Conf. Ser. 597012023 (2015).

[4] E. Celeghini and M.A. del Olmo, J. Phys. Conf. Ser. 597012022 (2015).

[5] A. Bohm, The Rigged Hilbert Space and Quantum Mechanics (Springer-Verlag, Berlin, 1978).

[6] J.E. Roberts, Comm. Math. Phys. 3, 98-119 (1966).

[7] J.P. Antoine, J. Math. Phys. 10, 53-69 (1969).

[8] O. Melsheimer, J. Math. Phys. 15, 902-916 (1974).

[9] M. Gadella and F. Gómez, Found. Phys. 32, 815869 (2002).

[10] M. Gadella and F. Gómez, Int. J. Theor. Phys. 42, 2225-2254 (2003).

[11] M. Gadella, F. Gómez-Cubillo, Acta App. Math. 109 (3), 721-742 (2010).

[12] A. Bohm and M. Gadella, Dirac Kets, Gamow Vectors and Gelfand Triplets, Springer Lec. Notes in Phys. 348 (Springer, Berlin, 1989).

[13] O. Civitarese and M. Gadella, Phys. Rep. 396 41113 (2004).

[14] H.G. Feichtinger, AIP Conf. Proc., 1146, 189-228 (2009).

[15] G. Bellomonte, S. Di Bella and C. Trapani, J. Math. Annal. Appl. 411, 931-946 (2014).

[16] S. Di Bella and C. Trapani, Mediterr. J. Math. 13, 2011-2014 (2016).

[17] G. Bellomonte and C. Trapani, Zeitschrift fr Analyse und ihre Anwendungen 35 (3) 243-265 (2016).

[18] H. Chiba, Advances in Math. 273, 324-379 (2015).

[19] J. Heredia-Juesas, E. Gago-Ribas and P. VidalGarcía, "Application of the Rigged Hilbert Spaces into the Generalized Signals and Systems Theory: Practical Example" in PIERS Proceedings, (IEEE, New York, 2016).

[20] J.P. Antoine and C. Trapani, Axioms 8(2), 52 (2019).

[21] C. Trapani, S. Triolo and F. Tschinke, J. Four. Anal. Appl. 25, 2109-2140 (2019).

[22] E. Celeghini, M. Gadella and M.A. del Olmo, J. Math. Phys. 57072105 (2016).

[23] E. Celeghini, M. Gadella and M.A. del Olmo, J. Math. Phys. 59, 053502 (2018).

[24] E. Celeghini, M. Gadella, M.A. del Olmo, Acta Polytech. 57(6), 379-384 (2017).

[25] E. Celeghini, M. Gadella, M.A. del Olmo, Entropy 20, 816/14, doi:10.3390/e20110816 (2018).

[26] E. Celeghini, M. Gadella and M.A. del Olmo, Quantum Theory and Symmetries with Lie Theory and Its Applications in Physics vol 2, ed. V. Dobrev, pp 373-383 (Springer, Berlin, 2018).

[27] E. Celeghini, M. Gadella and M.A. del Olmo, J. Math. Phys. 60, 053502 (2019).

[28] E. Celeghini, M. Gadella and M.A. del Olmo, Axioms 8, 89, doi:10.3390/axioms8030089 (2019).

[29] E. Celeghini, M.A. del Olmo and M.A. Velasco, Jacobi polynomials as $S U(2,2)$ unitary irre- ducible representation in Integrability, Supersymmetry and Coherent States, CRM Series in Math. Phys., pp. 267-283, S. Kuru et al. (eds.) (Springer Nature Switzerland AG, Cham, 2019).

[30] F.W.J. Olver, D.W. Lozier, R. F. Boisvert and C. W. Clark, Eds, NIST Handbook of Mathematical Functions, (NITS and Cambridge Univ. Press, Cambridge, UK, 2010).

[31] W. Magnus, F. Oberhettinger and R.P. Soni, Formulas and Theorems for the Special Functions of Mathematical Physics, (Springer, Berlin, 1966).

[32] J.A. Calzada, J. Negro and M.A. del Olmo, J. Math. Phys. 47043511 (2006).

[33] F. Tremblay, A.V. Turbiner and P. Winternitz, J. Phys. A: Math. Theor. 42242001 (2009).

[34] E. Wigner, Zeitschrift für Physik 43, 624 (1927).

[35] L.C. Biedenharn and J.D. Louck, Angular Momentum in Quantum Mechanics (Addison- Wesley, Reading, 1981).

[36] D.I. Caruntu, J. Sound and Vibration 306, 467494 (2007).

[37] D.Tchiotsop, D. Wolf, V. Louis-Dorr and R. Husson, Proc.of the 29th Anual Int. Conf.of the IEEE EMBS 2007, pp.1863-1867( IEEE 2007).

[38] P. Karczmarek, D. Pylak and M. A.Sheshko, Applied Math. and Comput. 181, 694-707 (2006).

[39] M. M.Bahşi, A.K. Bahşi, M. Çevik and M. Sezer, Mathematical Sciences 10, 83-93 (2016).

[40] Ben-yu Guo, J. Math. Analysis and Appl. 243, 373-408 (2000).

[41] J. Shen and Li-Lian Wang, Commun. Comput. Phys, 5, 195-241 (2009).

[42] G. Szegö, Orthogonal Polynomials, Coloquium Publications 23 (Am. Math. Soc., Providence, 2003).

[43] R. Askey, Orthogonal Polynomials and Special Functions, Regional Conf. Ser. in Applied Math. 21 (SIAM, Philadelphia, 1975).

[44] A.O. Barut, J.R. Zeni and A Laufert, J.Phys. A: Math. Gen. 27, 6799-6805 (1994).

[45] M.A. del Olmo, M.A. Rodríguez and P. Winternitz, Fortschritte der Physik, 44, 91-125 (1996).

[46] M. Calixto and E. Pérez-Romero, J. Math. Phys. 55081706 (2014).

[47] G. Mack, Commun. Math. Phys. 55, 1-28 (1977).

[48] N. Jacobson, Lie algebras (Dover, New York, 1979).

[49] A. Pietsch, Nuclear Locally Convex Spaces (Springer, Berlin, 1972).

[50] M. Reed and B. Simon, Functional Analysis (Academic, New York, 1977)

[51] H.J. Weber and G.B. Arfken, Essential Mathematical Methods for Physicists (Elsevier, Amsterdam, 2004)

[52] G Hunter, P Ecimovic, I Schlifer, I M Walker, D Beamish, S Donev, M Kowalski, S Arslan and S Heck, J. Phys. A: Math. Gen. 32, 795-803 (1999).

[53] M. Abramovich, I.A. Stegun, Handbook of Mathematical Functions (10th printing), National Bu- 
reau of Standards Appl. Math. Ser. 55 (U.S. Government Printing Office, Washington, 1972).
[54] U. Haagerup and H. Schlichtkrull, Ramanujan J. 33, 227-246 (2014). 